\title{
Normal State Properties of Quantum Critical Metals at Finite Temperature
}

\author{
Avraham Klein® and Andrey V. Chubukov $\odot$ \\ School of Physics and Astronomy, University of Minnesota, Minneapolis, Minnesota 55455, USA \\ Yoni Schattner® \\ Department of Physics, Stanford University, California 94305-4013, USA \\ and Stanford Institute for Materials and Energy Sciences, \\ SLAC National Accelerator Laboratory and Stanford University, \\ Menlo Park, California 94025-7015, USA \\ Erez Berg \\ Department of Condensed Matter Physics, Weizmann Institute of Sciences, Rehovot 7610001, Israel
}

(Received 24 March 2020; revised 4 June 2020; accepted 8 July 2020; published 8 September 2020)

\begin{abstract}
We study the effects of finite temperature on normal state properties of a metal near a quantum critical point to an antiferromagnetic or Ising-nematic state. At $T=0$, bosonic and fermionic self-energies are traditionally computed within Eliashberg theory, and they obey scaling relations with characteristic power laws. Corrections to Eliashberg theory break these power laws but only at very small frequencies. Quantum Monte Carlo (QMC) simulations have shown that, already at much larger frequencies, there are strong systematic deviations from these predictions, casting doubt on the validity of the theoretical analysis. We extend Eliashberg theory to finite $T$ and argue that in the $T$ range accessible in the QMC simulations above the superconducting transition, the scaling forms for both fermionic and bosonic self-energies are quite different from those at $T=0$. We compare finite $T$ results with QMC data and find good agreement for both systems. We argue that this agreement resolves the key apparent contradiction between the theory and the QMC simulations.
\end{abstract}

DOI: $10.1103 /$ PhysRevX.10.031053

Subject Areas: Condensed Matter Physics

Strongly Correlated Materials

Superconductivity

\section{INTRODUCTION}

Electron-boson models [1-4] have long been used to study the behavior of interacting fermions near a metallic quantum critical point (QCP). In these models, a specific channel of the electron-electron interaction is assumed to become critical at a QCP and is represented by a soft collective boson, while all other channels are assumed to be irrelevant to the low-energy dynamics near the QCP. Despite their simplicity, such models predict nontrivial correlation effects such as superconductivity and non Fermi-liquid behavior in the normal state [3-18]. Correlation effects are stronger in two dimensions (2D) than in 3D, consistent with the fact that most systems in which non Fermi-liquid behavior and high-temperature superconductivity have been observed, e.g., $\mathrm{Cu}$ - and Fe-based superconductors, are

Published by the American Physical Society under the terms of the Creative Commons Attribution 4.0 International license. Further distribution of this work must maintain attribution to the author(s) and the published article's title, journal citation, and DOI.
quasi-2D systems [4,19-26]. Two of the most-studied models of metallic quantum criticality are the spin-fermion model [4], where the boson describes fluctuations of an antiferromagnetic order parameter, and the Ising-nematic model [7], where the boson represents an order parameter, which breaks lattice rotational symmetry (a similar, but inequivalent behavior emerges in isotropic systems near, e.g., a Pomeranchuk instability in a $d$-wave channel [6]).

Both the spin-fermion and the Ising nematic models have been analyzed within the low-energy theory, which is termed Eliashberg theory (ET) due to its similarity with the Eliashberg theory of the electron-phonon interaction. The theory assumes that near a QCP, a soft collective boson is a slow mode compared to a dressed fermion. This effectively decouples the fermionic and bosonic degrees of freedom $[4,7,27,28]$ and makes the problem analytically tractable. In technical terms, in ET the fermionic selfenergy $\Sigma(k, \omega)$ is large, but it can be approximated by its value at the Fermi energy $\Sigma\left(k_{F}, \omega\right)$ and computed perturbatively at one-loop order. Higher-order corrections (commonly termed vertex corrections) are neglected by the argument that in the processes giving rise to vertex 
corrections, fermions oscillate at frequencies near the bosonic mass shell, which are far away from their own mass shell. In addition, the integration over internal momenta in the one-loop diagram for the fermionic selfenergy factorizes into the one transverse to the Fermi surface (FS), which involves only fermionic degrees of freedom, and the one parallel to the FS, involving only bosonic degrees of freedom. In this situation, characteristic momentum deviations from the FS are small, and the integration can be carried out by linearizing the fermionic dispersion near $k_{F}$. By the same argument, the bosonic selfenergy is also computed perturbatively, at one-loop order.

The decoupling of the fermions and bosons leads to fermionic self-energy, which depends much more strongly on the frequency $\omega$ than on deviation of the momentum from $k_{F}$ transverse to the FS. The magnitude of the $\omega$ dependent self-energy in turn depends on the location of $k_{F}$ on the FS. In the Ising-nematic model, the Eliashberg selfenergy scales as $\Sigma\left(\omega, \mathbf{k}_{F}\right) \sim \omega_{\mathrm{INM}}^{1 / 3} \omega^{2 / 3}$ over the whole FS, except at special points (cold spots), where Fermiliquid behavior survives. In the spin-fermion model, the Eliashberg self-energy scales as $\Sigma\left(\omega, \mathbf{k}_{F}\right) \sim \omega_{\mathrm{SFM}}^{1 / 2} \omega^{1 / 2}$ at special FS points (hot spots), connected by a momentum vector corresponding to antiferromagnetic order, while everywhere else on the FS, the self-energy has a Fermiliquid form at the lowest frequencies and crosses over to $\omega^{1 / 2}$ behavior at a characteristic frequency proportional to the deviation from a hot spot. The $\omega_{\mathrm{SFM}}$ and $\omega_{\mathrm{INM}}$ are characteristic frequencies, which we discuss below. Both remain finite at a QCP.

The validity of the ET in the case where a soft boson is a collective mode of fermions is a more tricky issue than for the original Eliashberg theory of superconductivity, where the boson is an independent degree of freedom (a phonon). For that theory, the applicability condition is the smallness of the ratio $\omega_{0} / E_{F} \sim v_{0} / v_{F}$-where $\omega_{0}$ is the characteristic phonon frequency and $v_{0}$ is the corresponding boson velocity (the dressed Debye frequency and sound velocity for an acoustic phonon) and $E_{F}, v_{F}$ are the Fermi energy and velocity (this allows one to factorize the momentum integration) - and the smallness of the ratio $\alpha^{2} /\left(\omega_{0} E_{F}\right)$, where $\alpha$ is the effective fermion-boson coupling (this allows one to neglect vertex corrections). The last condition is not satisfied when $\omega_{0}$ vanishes, but for large enough $E_{F}$, it holds in a wide range of $\omega_{0}$ (Ref. [29]). When the boson is a collective mode in the spin or charge channel, its bare velocity is of the order of $v_{F}$, so at the bare level, the ET is inapplicable. However, in both the spin-fermion and Ising-nematic models, a dressed collective boson is Landau overdamped due to the decay into particle-hole pairs. This case opens up a possibility that at low energies, a dressed boson becomes slow compared to a dressed fermion; i.e., ET becomes applicable as an effective theory, which describes dressed bosons and fermions at low energies. For the Ising-nematic model, the Landau-overdamped boson is slow compared to dressed fermions by $\left(\omega / \omega_{\text {INM }}\right)^{1 / 3}$ (Refs. [3,6,7,28,30]), which justifies factorization of momentum integration leading to $\omega^{2 / 3}$ scaling at a QCP. Vertex corrections diverge at a QCP, when calculated with free fermions, but remain finite within the effective ET. The lowest-order vertex correction is of order one, but it can be made parametrically small if one extends the theory to $N \gg 1$ fermionic flavors [3,28]. However, at two loops, there are unavoidable logarithmical singularities for both $\Sigma\left(\mathbf{k}_{F}, \omega\right)$ and $\Sigma(\mathbf{k}, 0)$ (Refs. [8,31]). These logarithms come from special "planar diagrams," which describe hidden 1D processes with a momentum transfer of either 0 or $2 k_{F}$ (Ref. [30]). Logarithmical corrections were also reported for a bosonic propagator in four-loop calculations [32,33]. These logarithms are not accounted for in the effective ET [34]. For the spin-fermion model, the velocities of dressed fermions and bosons are comparable; i.e., corrections to $\Sigma_{\mathrm{SFM}}(\omega)=\omega^{1 / 2} \omega_{\mathrm{SFM}}^{1 / 2}$ are of order one. This can be cured by extending the theory to $N \gg 1$ fermionic flavors, in which case, the corrections to factorization are small in $1 / N$. The $k$-dependent self-energy and vertex are also small in $1 / N$. However, just like in the Ising-nematic model, there are logarithmical corrections to the effective ET. Moreover, in the spin-fermion model, logarithms appear already in oneloop $\Sigma(k)$ and vertex corrections $[4,9,12,35]$.

This analysis shows that for both models, the effective ET becomes invalid below some characteristic frequency, at which logarithmic corrections become of order one. However, for the breakdown of ET to occur, this frequency must be larger than superconducting $T_{c}$; otherwise, the logarithmic singularities will be cut off by the opening of a gap due to superconductivity. In the spin-fermion model, $T_{c}$ is generally of order $\omega_{\mathrm{SFM}}$, and in the Ising-nematic model, $T_{c} \sim \omega_{\text {INM }}$ (Refs. [4,5,17,36,37]). At such frequencies, some calculations show [38] that corrections to ET may be small numerically, in which case the effective ET should remain valid, at least qualitatively.

The validity of the effective ET at a QCP has recently been tested in a series of sign-free quantum Monte Carlo $(\mathrm{QMC})$ simulations of both the spin-fermion and Isingnematic models $[11,39-46]$. Such simulations are numerical experiments that test effective models of quantumcritical metals $[45,47,48]$. QMC data were taken at temperatures above $T_{c}$, where ET is expected to work. Analysis of the QMC data revealed that some properties, most strikingly the superconducting $T_{c}$ of the spin-fermion model, agreed well with predictions of ET [42]. However, other properties showed systematic deviations from ET. In particular, for both the spin-fermion and Ising-nematic models, fermionic self-energies in the normal state, extracted from QMC, do not show the power-law forms, expected from the theory, and appear to saturate at a finite value even at the smallest fermionic Matsubara frequency $\omega=\pi T$. In addition, the bosonic self-energy in the Isingnematic model does not show the expected $\Omega / q$ scaling of a Landau-overdamped boson. The apparent contradiction 
with the numerical experiments has cast into doubt the validity of ET.

In this work, we argue that the discrepancies of the QMC data with the ET can be reconciled by properly accounting for finite-temperature effects within appropriately modified ET (MET), which, we argue, differs qualitatively from the ET at $T=0$ (we will keep the notation ET for the $T=0$ Eliashberg theory). Several previous works have studied the validity of ET at finite temperature, as well as finitetemperature effects within the theory [4,49-52]. They showed that the central effect of finite temperature is to split the fermionic self-energy into the sum of two parts,

$$
\Sigma\left(\omega_{m}\right)=\Sigma_{T}\left(\omega_{m}\right)+\Sigma_{Q}\left(\omega_{m}\right),
$$

where $\Sigma_{T}\left(\omega_{m}\right)$ is the thermal contribution, coming from the static bosonic propagator, $\Sigma_{Q}\left(\omega_{m}\right)$ comes from the dynamic propagator, and, in this work, we define $\Sigma\left(\omega_{m}\right)$ as a function of a fermionic frequency on the Matsubara axis (the one that can be directly compared with QMC results). We make a detailed comparison of our results with previous work in Sec. III, and here, for clarity, we present our central conclusions, which are in broad agreement with these earlier works. We argue that at finite temperature, one has to go beyond perturbation theory and compute fermionic $\Sigma(\omega)$ and bosonic $\Pi(\Omega)$ self-consistently and without factorization of momentum integration. More precisely, the thermal piece $\Sigma_{T}$ needs to be calculated self-consistently without factorizing the momentum integration. The dynamical $\Sigma_{Q}$ does not need to be computed self-consistently, but at $\omega_{m} \sim T$, one cannot factorize the momentum integration for this term as well. In addition, in the specific case of the Ising-nematic model, $\Pi\left(\Omega_{m}\right)$ needs to be calculated self-consistently as well.

We show that at finite $T$, there are two characteristic scales, a larger one and a smaller one. The larger scale, $\omega_{T}$, is the same for both the spin-fermion and Ising-nematic models, and it is

$$
\omega_{T} \sim \sqrt{\bar{g} T \log \frac{\sqrt{\bar{g} T}}{v_{F} M}}
$$

where $\bar{g}$ is the effective fermion-boson coupling (defined below), $v_{F}$ is the Fermi velocity, and $M$ is the bosonic thermal mass, which we treat here as a small parameter, consistent with the QMC results. The smaller scale is

$$
\omega_{T}^{\prime} \sim \omega_{T} \begin{cases}\left(\frac{T}{\bar{g}}\right)^{1 / 2} & \text { spin-fermion model } \\ \frac{T}{E_{F}} \log \frac{\sqrt{\bar{g} T}}{v_{F} M} & \text { Ising-nematic model. }\end{cases}
$$

We assume $T \ll \bar{g}, E_{F}$, such that in both models $\omega_{T}^{\prime} \ll \omega_{T}$.

At the smallest Matsubara frequencies, $\omega_{m} \ll \omega_{T}^{\prime}$, the two components of the self-energy have the form

$$
\Sigma_{T}\left(\omega_{m}\right) \sim \omega_{T}, \quad \Sigma_{Q}\left(\omega_{m}\right) \sim \frac{\omega_{m}}{\omega_{T}} .
$$

We call this regime strongly thermal. At high Matsubara frequencies, $\omega_{m} \gg \omega_{T}$, the self-energy components have the form

$$
\begin{aligned}
& \Sigma_{T}\left(\omega_{m}\right) \sim \frac{\omega_{T}^{2}}{\omega_{m}}, \\
& \Sigma_{Q}\left(\omega_{m}\right) \sim \begin{cases}\omega_{m}^{1 / 2} & \text { spin-fermion model } \\
\omega_{m}^{2 / 3} & \text { Ising-nematic model. }\end{cases}
\end{aligned}
$$

We call this regime almost critical. In between these two regimes, i.e., at $\omega_{T}^{\prime}<\omega_{m}<\omega_{T}$, the system behavior is rather complex, and there is no particular scaling behavior for both $\Sigma_{T}\left(\omega_{m}\right)$ and $\Sigma_{Q}\left(\omega_{m}\right)$. We argue that most of the QMC data in Refs. [11,39,40] fall into this intermediate frequency region.

We compute $\Sigma_{T}\left(\omega_{m}\right)$ and $\Sigma_{Q}\left(\omega_{m}\right)$ within MET and compare the result with QMC data. For both the spinfermion and Ising-nematic models, we show that $\Sigma\left(\omega_{m}\right)=$ $\Sigma_{T}\left(\omega_{m}\right)+\Sigma_{Q}\left(\omega_{m}\right)$ agrees with QMC results. The agreement holds for both the magnitude of $\Sigma\left(\omega_{m}\right)$ and its dependence on frequency. We show that in the temperature range of the QMC simulations, thermal effects are essential, and $\Sigma_{\mathrm{SFM}}\left(\omega_{m}\right)$ and $\Sigma_{\mathrm{INM}}\left(\omega_{m}\right)$, obtained in the MET, are quite flat functions of frequency. The bosonic propagator $D\left(\Omega_{m}, q\right)$, obtained within MET, also agrees with QMC result. This agreement is particularly significant for the Ising-nematic model, where QMC shows that the frequency dependence of $D^{-1}\left(\Omega_{m}, q\right)$ is proportional to $\left|\Omega_{m}\right|$ rather than $\left|\Omega_{m}\right| / q$, which is expected for Landau damping. The absence of $\left|\Omega_{m}\right| / q$ scaling at the smallest $q$ is due to the fact that the Ising-nematic order parameter is not a conserved quantity, but the near absence of the $q$ dependence over a wide range of $q$ is chiefly the consequence of the flatness of $\Sigma\left(\omega_{m}\right)$ in the $T$ range probed by QMC.

For comparison, we also compute $\Sigma_{T}\left(\omega_{m}\right)$ and $\Sigma_{Q}\left(\omega_{m}\right)$ using the same equations as in the MET, but we integrate over the internal fermionic momenta in the full Brillouin zone (i.e., compute the self-energy without linearizing the fermionic dispersion near the FS). We call this the lattice theory (LT). We show that the forms of the self-energies in MET and LT are qualitatively similar, but with differences in the details. We note, in this regard, that while in both MET and LT one neglects vertex corrections and extracts $\Sigma$ from a self-consistent analysis, $\Sigma$ in LT contains an additional piece coming from high-energy fermions, with energies of the order of the bandwidth. Because vertex corrections also predominantly come from high-energy fermions, by comparing the self-energies in MET and LT to the one extracted from QMC, one can verify whether there is at least a partial cancellation between the vertex corrections and contributions from high-energy fermions to 
the self-energy. We show that QMC data agree somewhat better with MET than with LT, particularly for the Isingnematic model. This result suggests that there may be some cancellation between different contributions from highenergy fermions.

Our results demonstrate that (a) current QMC data are consistent with the MET (i.e., ET, properly extended to finite $T$ ) and that (b) the comparison between MET, LT, and QMC provides a framework to identify the strength of the correlation effects that are not captured by the low-energy MET. We hope that our results will provide useful input, both to further analytical work and for analysis of upcoming numerical results.

The rest of the paper is organized as follows. In Sec. II, we introduce the spin-fermion and Ising-nematic models and review the low-temperature predictions of ET. In Sec. III, we discuss the modified ET at a finite temperature, which takes into account thermal fluctuations. In Sec. IV, we briefly review the lattice models that we use in the LT calculation of the self-energy. Finally, in Sec. V, we compare our results to the QMC data. We present our conclusions in Sec. VI.

\section{ELIASHBERG THEORY FOR THE SPIN-FERMION AND ISING-NEMATIC MODELS}

\section{A. Models}

Both the spin-fermion and the Ising-nematic models are described within a single framework. We assume a 2D system of spinful fermions coupled to a single bosonic field. The effective field theory is

$$
\mathcal{S}=\mathcal{S}_{f}+\mathcal{S}_{b}+\mathcal{S}_{I}
$$

It is the sum of three terms-a fermionic action $\mathcal{S}_{f}$, a bosonic action $\mathcal{S}_{b}$, and an interaction $\mathcal{S}_{I}$. The fermionic action is

$$
\mathcal{S}_{f}=\int d \tau \sum_{\mathbf{p}, \sigma} \psi_{\sigma}^{\dagger}(\mathbf{p}, \tau)\left(\partial_{\tau}-\epsilon_{\mathbf{k}}\right) \psi_{\sigma}(\mathbf{p}, \tau),
$$

where $\sigma$ sums over fermion spins and $\epsilon_{\mathbf{k}}$ is the band dispersion. Figure 1 schematically depicts the FS for the spin-fermion and Ising-nematic models. The bosonic action is

$$
\mathcal{S}_{b}=\int d \tau \sum_{\mathbf{q}, j} \phi_{j}(\mathbf{q}, \tau) \frac{M_{0}^{2}+|\mathbf{q}-\mathbf{Q}|^{2}}{D_{0}} \phi_{j}(\mathbf{q}, \tau),
$$

where $j=1, \ldots, N_{b}$ sums over $N_{b}$ boson components, $D_{0}$ is a constant with units of (area $\times$ energy $)^{-1}$, and $M_{0}$ measures the distance of the bosons from the QCP. We treat $M_{0}=M_{0}(T)$ as a parameter, and our results do not explicitly depend on the temperature dependence of $M_{0}$,

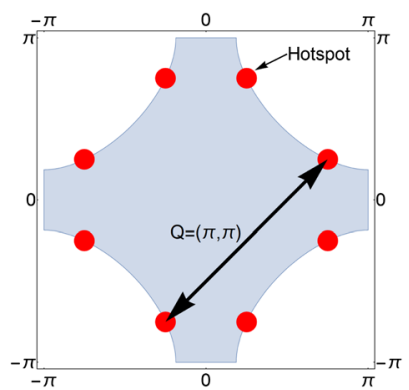

(a)

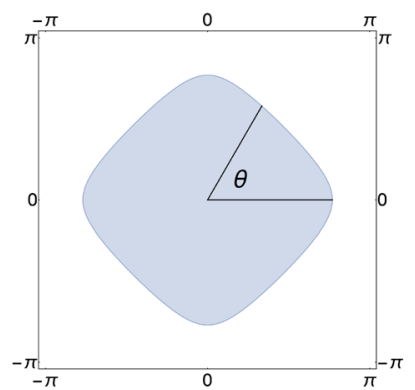

(b)
FIG. 1. FS structure of the spin-fermion and Ising-nematic metals. (a) FS structure of the spin-fermion model, which has four hole pockets, with "hotspots" connected by the AF $(\pi, \pi)$ vector. (b) The electron FS in the nematic problem, with the FS angle $\theta$ denoted in the figure.

which can have, e.g., a Curie-Weiss form, $M_{0}^{2} \propto T-T_{0}$. Note that $\mathbf{Q}$ is the momentum at which a collective boson softens at a QCP: $\mathbf{Q}_{\mathrm{SFM}}=(\pi / a, \pi / a)$ and $\mathbf{Q}_{\mathrm{INM}}=0(a$ is the interatomic spacing). The boson is assumed to have no bare dynamics and acquires it solely from the interaction with fermions. (More accurately, the assumption is that the bare boson dynamics exists, but it is irrelevant compared to the acquired one.) The interaction is described by

$$
\begin{aligned}
\mathcal{S}_{I}= & g \int d \tau \sum_{\substack{\mathbf{q} \sigma^{\prime} \\
j \sigma \sigma^{\prime}}} \phi_{j}(\mathbf{q}) t_{\sigma \sigma^{\prime}}^{j} f(\mathbf{p}) \\
& \times \psi_{\sigma}^{\dagger}\left(\mathbf{p}-\frac{\mathbf{q}}{2}\right) \psi_{\sigma^{\prime}}\left(\mathbf{p}+\mathbf{Q}+\frac{\mathbf{q}}{2}\right),
\end{aligned}
$$

where $g$ is the coupling constant, and $t_{\sigma \sigma^{\prime}}^{j}, f(\mathbf{p})$ express the spin and momentum form factors of the interaction.

Spin-fermion model.-In the spin-fermion model, the bosons represent spin fluctuations, so $\phi$ has up to three components, $t^{j}=\tau^{j}$ are Pauli matrices, and $f(\mathbf{p}) \approx 1$. The momentum $\mathbf{Q}=(\pi / a, \pi / a)$ couples $N=8$ discrete points on the FS, in sets of two [see Fig. 1(a)]. Consequently, the most relevant fermionic degrees of freedom are the ones near these "hot spots." The interaction between hot fermions and a $(\pi / a, \pi / a)$ boson has the form

$$
\begin{aligned}
\mathcal{S}_{I}^{\mathrm{SFM}} & =\int d \tau g \sum_{\mathbf{q} \mathbf{p} l \sigma \sigma^{\prime}} \vec{\phi}(\mathbf{Q}+\mathbf{q}) \\
& \cdot \psi_{l \sigma}^{\dagger}\left(\mathbf{p}-\frac{\mathbf{q}}{2}\right) \vec{\tau}_{\sigma \sigma^{\prime}} \psi_{l \sigma^{\prime}}\left(\mathbf{p}+\mathbf{Q}+\frac{\mathbf{q}}{2}\right),
\end{aligned}
$$

where $l=1, \ldots, N$ label the hot spots.

Ising-nematic model.- In the Ising-nematic model, the boson represents a nematic deformation along one of two symmetry axes. Accordingly, $\phi$ is a scalar. Because $\mathbf{Q}=0$, particles on the entire FS participate in the critical dynamics, and the interaction has the form 


$$
\mathcal{S}_{I}^{\mathrm{INM}}=\int d \tau g \sum_{\mathbf{q}, \mathbf{p}, \sigma} \phi(\mathbf{q}) \psi_{\sigma}^{\dagger}(\mathbf{p}-\mathbf{q} / 2) f(\mathbf{p}) \psi_{\sigma}(\mathbf{p}+\mathbf{q} / 2),
$$

where $f(\mathbf{p})=\left(\bar{k}_{F}\right)^{-2}\left(p_{x}^{2}-p_{y}^{2}\right)$ encodes the nematic form factor and $\bar{k}_{F}=(2 \pi)^{-1} \int d \theta k_{F}(\theta)$ is the FS-averaged Fermi vector, where $\theta$ traces out a direction on the FS [see Fig. 1(b)]. Near the FS, $f(\mathbf{p})$ can be approximated as a function of $\theta: f(\theta)=\left(k_{F}(\theta) / \bar{k}_{F}\right)^{2} \cos 2 \theta$.

\section{B. Review of diagrammatic theory}

We now briefly review diagrammatic perturbation theory for the two models. The dynamics of fermions and bosons is encoded in their self-energies $\Sigma(\omega, \mathbf{p})$ and $\Pi(\Omega, \mathbf{q})$ (the latter is also called a polarization bubble). We consider the self-energies on the Matsubara axis, where $\omega=\omega_{m}=2 \pi T(m+1 / 2)$ and $\Omega=\Omega_{m}=2 \pi T m$. As we noted in the Introduction, we compare the results on the Matsubara axis with the QMC data. We use Latin letters to denote frequency-momentum 3-vectors, $q=\left(\Omega_{m}, \mathbf{q}\right)$, $k=\left(\omega_{m}, \mathbf{k}\right)$. The self-energies are related to bosonic and fermionic propagators as

$$
G(k)=\left(i \omega_{m}+i \Sigma(k)-\epsilon(\mathbf{k})\right)^{-1}
$$

and

$$
D(q)=D_{0}\left(M_{0}^{2}+(\mathbf{q}-\mathbf{Q})^{2}+\Pi(q)\right)^{-1} .
$$

Both self-energies are represented by a diagrammatic series in the effective coupling $\bar{g}=g^{2} D_{0}$. We assume that $\bar{g}$ is small compared to the Fermi energy $E_{F}$. Then, we can safely restrict to the lowest-order expansion in $\mathbf{q}-\mathbf{Q}$ in Eq. (13) and restrict $\mathbf{k}$ in Eq. (12) to be near the FS.

The diagrammatic expressions for $G(k)$ and $\Pi(q)$ are presented in Fig. 2. Thick lines in this figure are fully dressed $G(k)$ and $D(q)$, and the solid triangles are fully renormalized vertices. In the ET, as well as in several other computational methods, e.g., fluctuation-exchange (FLEX) and dynamical mean-field approximations, vertex corrections are neglected. As stated in the Introduction, for the cases when bosons are collective modes of fermions, there is no small parameter to justify this step, unless one extends the theory to, e.g., a large number of fermionic flavors. Furthermore, vertex corrections coming from low-energy fermions are logarithmically singular. However, in both the spin-fermion and Ising-nematic models, these corrections are rather small numerically at frequencies above superconducting $T_{c}$, and we proceed by neglecting them without further discussion. There are also vertex corrections coming from high-energy fermions (the ones with energies of order bandwidth). These corrections are regular and, to a first approximation, can be absorbed into the renormalization of the coupling $\bar{g}$.

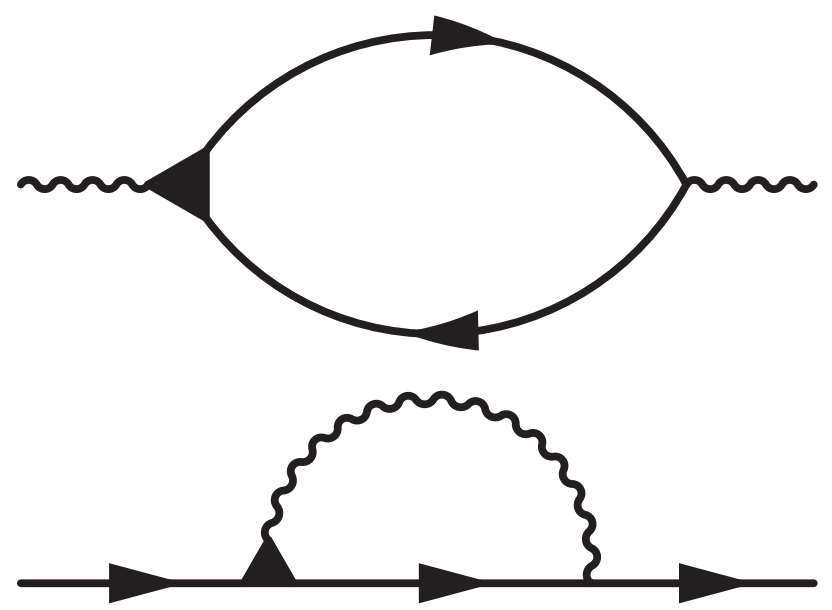

FIG. 2. Diagrammatic expressions for $\Pi(q)$ (top diagram) and $\Sigma(k)$ (bottom diagram). Solid lines are fully dressed fermion propagators $G(k)$, wavy lines are fully dressed boson propagators $D(q)$, and solid triangles are fully dressed vertex functions.

Without vertex corrections, $\Sigma(\mathbf{k})$ and $\Pi(\mathbf{q})$ are selfconsistently expressed as

$$
i \Sigma(k)=N_{b} \bar{g} T \sum_{n} \int \frac{d^{2} p}{(2 \pi)^{2}} f^{2}(\mathbf{k}+\mathbf{p} / 2) G(p+k) D(p),
$$

$\Pi(q)=2 \bar{g} T \sum_{n} \int \frac{d^{2} p}{(2 \pi)^{2}} f^{2}(\mathbf{p}+\mathbf{q} / 2) G(p+q) G(p)$.

Here, the factor $b$ in $\Sigma$ comes from summation over boson components, and the factor 2 in $\Pi$ comes from spin summation.

The rhs of Eqs. (14) and (15) contain integrals over fermionic momenta. In principle, these integrals are over the whole Brillouin zone. In the ET for fermion-boson models, it is further assumed that the contributions from high-energy fermions to the rhs of Eqs. (14) and (15) can be absorbed into $\bar{g}$. This renormalization of the coupling is in addition to the ones discussed above due to high-energy static vertex corrections. Therefore, we restrict ourselves to contributions from only low-energy fermions treating $\bar{g}$ in Eqs. (14) and (15) as the effective, dressed couplings, which include both vertex corrections and the contributions from high-energy fermions to the rhs of Eqs. (14) and (15). The dressed couplings in these two equations are generally not equal, but the nonequivalence only affects the numerical factors in the formulas below; for simplicity, we keep the same $\bar{g}$ in the equations for $\Sigma(k)$ and $\Pi(q)$.

The static contribution to the polarization $\Pi(q)$ serves to renormalize the parameters $D_{0}$ and $M_{0}$ in Eq. (13). In particular, it shifts the bosonic mass to

$$
M^{2}=M_{0}^{2}-\Pi\left(\Omega_{m}=0, \mathbf{q}=\mathbf{Q}\right) .
$$


The dynamical contribution, which gives rise to Landau damping of a boson, can be obtained most straightforwardly by first integrating in Eq. (15) over momentum and then summing up over Matsubara frequencies [53]. We shift the incoming bosonic momentum to the vicinity of the ordering vector $\mathbf{q} \rightarrow \mathbf{Q}+\mathbf{q}$ and symmetrize Eq. (15) by shifting $\mathbf{p} \rightarrow \mathbf{p}-\mathbf{q} / 2$. We linearize the fermionic dispersion near the FS as $\epsilon(\mathbf{p}+\mathbf{Q}+\mathbf{q}) \approx \epsilon+\mathbf{v}_{F} \cdot \mathbf{q}$, where $\mathbf{v}_{F}$ is the Fermi velocity at $\mathbf{p}_{F}+\mathbf{Q}$ and $\mathbf{p}_{F}(\theta)=$ $k_{F}(\theta)(\cos \theta, \sin \theta)$ is the Fermi vector with FS angle $\theta$. We then replace the momentum integration by an integral over the dispersion, as $d^{2} p \approx d \theta \nu_{F}(\theta) d \epsilon$, where $\nu_{F}(\theta)=$ $k_{F}(\theta) / v_{F}(\theta)$ is the density of states (DOS). We integrate over $\epsilon$ and obtain

$$
\Pi\left(\mathbf{Q}+\mathbf{q}, \Omega_{m}\right)=2 i \bar{g} T \sum_{n} \int \frac{d \theta}{2 \pi} \nu_{F}(\theta) f^{2}(\theta) \frac{\Theta\left(\omega_{n}+\Omega_{m}\right)-\Theta\left(\omega_{n}\right)}{i\left(\Omega_{m}+\Sigma\left(\omega_{n}+\Omega_{m}\right)-\Sigma\left(\omega_{n}\right)\right)-\left(\epsilon\left(\mathbf{p}_{F}(\theta)+\mathbf{Q}+\mathbf{q} / 2\right)-\epsilon\left(\mathbf{p}_{F}(\theta)-\mathbf{q} / 2\right)\right)} .
$$

In Eq. (17), $\epsilon\left(\mathbf{p}_{F}(\theta)+\cdots\right)$ should be understood as the dispersion linearized near the FS.

The fermionic self-energy for a fermion on the FS, i.e., for $|\mathbf{k}|=k_{F}\left(\theta_{k}\right)$, is obtained by linearizing the dispersion of an internal fermion near the FS as $\epsilon(\mathbf{k}+\mathbf{Q}+\mathbf{p}) \approx \epsilon(\mathbf{k})+v_{F}\left(\theta_{k+Q}\right)|\mathbf{p}| \cos \left(\theta-\theta_{k+Q}\right)$, where $\theta_{k+Q}$ is the FS angle of $\mathbf{k}+\mathbf{Q}$ and $\theta$ is the angle of $\mathbf{p}$. Evaluating the angular integral, we obtain

$$
\Sigma(k) \approx N_{b} \bar{g} T \sum_{n} \int \frac{|\mathbf{p}| d p}{2 \pi} \frac{\sigma\left(\omega_{n}\right)}{\sqrt{\left(\omega_{n}+\Sigma\left(\omega_{n}\right)\right)+\left[v_{F}\left(\theta_{k+Q}\right)\right]^{2}|\mathbf{p}|^{2}}} \frac{f^{2}\left(\theta_{k+Q / 2}\right)}{M^{2}+|\mathbf{p}|^{2}+\Pi\left(|\mathbf{p}| \hat{n}\left(\theta_{k+Q}\right), \omega_{m}-\omega_{n}\right)},
$$

where $\hat{n}\left(\theta_{k+Q}\right)=\left.\left[\left(-\mathbf{v}_{F y}, \mathbf{v}_{F x}\right) / v_{F}\right]\right|_{\theta=\theta_{k+Q}}$ is a unit vector pointing parallel to the $\mathrm{FS}$ at the angle $\theta_{k+Q}$. Here, $\sigma(x)$ is the sign function.

Equations (17) and (18) form a self-consistent set of equations for the low-energy dynamics of fermions near the FS. We next review how they are rendered analytically tractable in the ET.

\section{Eliashberg theory at $\boldsymbol{T}=0$}

The main technical simplification step in ET $[3,4,7,28,49,54]$ is the additional assumption that at $T=0$ the typical momentum transfer of a fermion near its mass shell $v_{F}\left(|\mathbf{k}|-k_{F}\right) \sim \omega+\Sigma(\omega)$ is much smaller than the typical momentum transfer of a boson, i.e.,

$$
|\omega+\Sigma(\omega)| \ll v_{F}|\mathbf{q}|,
$$

where $\omega$ is a typical fermionic frequency and $|\mathbf{q}|$ is a typical bosonic momentum. As noted above, this condition allows one to factorize the momentum interaction between directions along and transverse to the FS. In addition, the ET assumes that relevant frequencies are much smaller than $E_{F}$, and it integrates over momenta in infinite limits.

We first show the results within the ET and then discuss it's validity for the spin-fermion and Ising-nematic models.

We begin with the bosonic self-energy. In Eq. (17), the dominant contributions come from regions where $\delta \epsilon=\epsilon(\mathbf{p}+\mathbf{Q}+\mathbf{q} / 2)-\epsilon(\mathbf{p}-\mathbf{q} / 2)$ vanishes. These regions are the source of the different bosonic dynamics for the spin-fermion and Ising-nematic models. In the spin-fermion model, $\delta \epsilon$ vanishes near the hotspots as $\delta \epsilon \approx v_{F}\left(\theta_{h s}\right) Q_{h s} \sin \left(\theta-\theta_{h s}\right)$, where $\theta_{h s}$ is the FS angle of a hotspot and $Q_{h s}=k_{F}\left(\theta_{h s}\right)\left|\mathbf{v}_{F y}^{2}-\mathbf{v}_{F x}^{2}\right| /\left[v_{F}\left(\theta_{h s}\right)\right]^{2}$. Expanding near the hotspots, replacing the Matsubara sum by an integral, and integrating, we obtain

$$
\Pi^{\mathrm{SFM}}\left(\Omega_{m}\right) \approx \bar{g} \frac{\nu_{F}\left(\theta_{h s}\right) N}{2 \pi} \frac{\left|\Omega_{m}\right|}{v_{F}\left(\theta_{h s}\right) Q_{h s}} .
$$

In the Ising-nematic model, $\delta \epsilon \approx v_{F}(\theta) q \cos \left(\theta-\theta_{q}\right)$ depends strongly on $|\mathbf{q}|, \theta_{q}$, and it is dominated by $\theta \approx \theta_{q} \pm \pi / 2$, yielding

$$
\Pi^{\mathrm{INM}}\left(\Omega_{m}, \mathbf{q}\right) \approx \bar{g} \frac{\nu_{F}\left(\theta_{q}\right)}{\pi} f^{2}\left(\theta_{q}\right) \frac{\left|\Omega_{m}\right|}{v_{F}\left(\theta_{q}\right)|\mathbf{q}|} .
$$

In Eq. (21), we replaced $\theta_{q} \pm \pi / 2$ in the arguments of $\nu_{F}, f$ with $\theta_{q}$, utilizing the $\mathrm{C} 4$ symmetry of the system. We emphasize that, in both cases, the result does not depend on fermionic $\Sigma\left(\omega_{m}\right)$. This is because, in ET, the polarization bubble is the convolution of two DOS's, and each DOS $\nu(\omega)=-\left(\nu_{F} / \pi\right) \operatorname{Im} \int d \mathbf{k} G(\omega, \mathbf{k})=\nu_{F} \sigma(\omega)$ is independent of $\Sigma(\omega)$.

We now turn to the fermionic self-energy. In Eq. (18), we can approximate $\left(\sqrt{\left(\omega_{m}+\Sigma\left(\omega_{m}\right)\right)^{2}+v_{F}^{2}|\mathbf{p}|^{2}}\right)^{-1} \approx\left(v_{F}|\mathbf{p}|\right)^{-1}$ because of the assumption of ET in Eq. (19). This term then contributes a factor proportional to $|\mathbf{p}|^{-1}$ to the integral, which reduces the effective dimensionality of the $\mathbf{p}$ integral and renders it one dimensional. Physically, this result means that the boson momentum $\mathbf{p}$ is confined to be parallel to the FS. Then, the momentum and frequency 
integrals are straightforward. For the spin-fermion model at the QCP $M=0$, we obtain

$$
\Sigma^{\mathrm{SFM}}\left(\omega_{m}\right) \approx \omega_{\mathrm{SFM}}^{1 / 2}\left|\omega_{m}\right|^{1 / 2} \sigma\left(\omega_{m}\right)
$$

where $\omega_{\mathrm{SFM}}=\left[N_{b}^{2} \bar{g} Q_{h s} / 2 \pi N k_{F}\left(\theta_{h s}\right)\right]$, while for the Isingnematic model, we get

$$
\Sigma^{\mathrm{INM}}\left(\omega_{m}\right) \approx \omega_{\mathrm{INM}}^{1 / 3}\left|\omega_{m}\right|^{2 / 3} \sigma\left(\omega_{m}\right),
$$

where $\omega_{\mathrm{INM}}=\left[\bar{g}^{2} f^{4}\left(\theta_{q}\right) / 8 \pi^{2} 3^{3 / 2} \nu_{F}\left(\theta_{q}\right) v_{F}^{2}\left(\theta_{q}\right)\right]$.

Now, we check the justification of the assumption we made, Eq. (19). First, consider the spin-fermion model. In both Eqs. (17) and (18), the Heaviside and sign functions limit internal Matsubara frequencies to be on the order of the external frequency. As we discussed in the Introduction, both the non-Fermi-liquid behavior and superconductivity in the spin-fermion model emerge at typical external frequencies on the order of $\omega \sim \omega_{\mathrm{SFM}} \sim \bar{g} / N$; see Eq. (22). In the bosonic self-energy, the typical bosonic momentum is $v_{F} Q_{h s} \sim E_{F}$, so the assumption is well justified as long as $\bar{g} \ll E_{F}$. On the other hand, in the fermionic self-energy, the typical boson momentum is $v_{F} p \sim \sqrt{\Pi\left(\omega_{\mathrm{SFM}}\right)} \sim \bar{g}$, so the bosonic and fermionic momenta are of the same order unless $N \gg 1$. In practice, $N=8$ of the spin-fermion model is sufficiently large that there is just a reduction of the scale $\omega_{\mathrm{SFM}}$ by a factor of order one. In the Ising-nematic model, the typical frequencies are of order $\omega \sim \omega_{\mathrm{INM}} \sim \bar{g}^{2} / E_{F} \ll E_{F}$, while the typical bosonic momenta are of order $v_{F} p \sim$ $\sqrt{\Pi\left(\omega_{\mathrm{INM}}\right)} \sim \bar{g}$. Thus, the Eliashberg condition is well obeyed for the Ising-nematic model.

We see that at low enough temperatures, all fermionic self-energies scale as power laws in $\omega_{m}$, with no additional temperature dependence. Therefore, the fermionic selfenergies at different temperatures should collapse onto one another and vanish at the lowest frequencies. Similarly, the bosonic self-energies in the spin-fermion model should collapse onto one another, while in the Ising-nematic model, they should scale with $1 /|\mathbf{q}|$. In the next section, we show that finite-temperature effects destroy these scaling properties.

\section{SELF-ENERGIES AT FINITE TEMPERATURES}

We now study the bosonic and fermionic self-energies at finite temperatures. We do not assume that the typical fermionic momentum is smaller than the bosonic momentum. In addition, we do not replace the Matsubara sum by a frequency integral, which means that we must account for the thermal piece that we discussed in the Introduction.
We start with the fermionic self-energy. We explicitly split the Matsubara sum into a thermal and a nonthermal part and rewrite $\Sigma$ as

$$
\Sigma\left(\omega_{m}\right) \approx \Sigma_{T}\left(\omega_{m}\right)+\Sigma_{Q}\left(\omega_{m}\right),
$$

where $\Sigma_{T}\left(\omega_{m}\right)$ contains only the $\omega_{m}=\omega_{n}$ term in the sum, and $\Sigma_{Q}\left(\omega_{m}\right)$ accounts for the nonthermal dynamical contributions. Here, $\Sigma_{T}$ should be understood as the thermal scattering rate of the fermions, akin to scattering due to static disorder [55].

Before proceeding to detailed calculations, we review previous work on the finite $T$ problem and discuss some general considerations. It has been understood for some time $[4,49,50,52]$ that finite temperature introduces an energy scale $\Sigma_{T} \sim \sqrt{\bar{g} T}$, as can be seen just from the prefactor of the thermal $m=n$ term in Eq. (18). The thermal scale only has a small effect on $\Sigma_{Q}$. To understand why, notice that the Eliashberg $T=0$ results, Eqs. (20)-(23), are actually the same as what would be obtained without self-consistency; i.e., within ET, the self-consistent self-energies are the same as the perturbative ones $[4,49]$. One may expect that for $\Sigma_{Q}\left(\omega_{m}\right)$ at sufficiently high $\omega_{m}$, typical internal momenta and energies obey Eq. (19), momentum integration can still be factorized, and the finite temperature $\Sigma_{Q}$ is still the same as the perturbative result, with some deviations at lower frequencies (we will discuss the precise scale below). For the thermal piece, the situation is different because the dynamic polarization $\Pi\left(\omega_{n}-\omega_{m}=0\right)=0$, implying an infrared divergence of the integral over bosonic momenta, arising from the unscreened long-range interaction, which is cut off by the thermal mass $M(T)[50,52,56]$. If $v_{F} M(T) \gg \sqrt{\bar{g} T}$, then momentum integration can still be factorized, typical bosonic momenta are about $v_{F} M$, and, as a result, $\Sigma_{T} \sim \bar{g} T / v_{F} M$, which is actually an analogous result to that of a Fermi liquid - the self-energy is just proportional to the effective static interaction $[4,49,51]$. If $v_{F} M(T) \lesssim \sqrt{\bar{g} T}$, momentum integration cannot be factorized, and the thermal mass cuts off the logarithmic divergence of the two-dimensional momentum integration. It has been noted [49,51] that when $M(T)$ is determined by the one-loop bosonic renormalization (the mode-mode coupling [2]), one expects $M(T) \sim \sqrt{\bar{g}^{2} T|\log T| / E_{F}}$. Therefore, except at exponentially low temperatures, the thermal self-energy must be calculated self-consistently and without factorization of momentum. Following the previous arguments, we assume that the thermal $\Sigma_{T}$ must be evaluated selfconsistently; however, $\Sigma_{Q}$ does not, and it only requires the self-consistent $\Sigma_{T}$ as an input. We first present the results and then discuss the validity of the approximation.

The self-energy $\Sigma_{T}$ is the solution of the self-consistent equation 


$$
\begin{aligned}
& \Sigma_{T}\left(\omega_{m}, \theta_{k}\right) \\
& \quad \approx N_{b} \bar{g} T \int \frac{|\mathbf{p}| d p}{2 \pi} \frac{\sigma\left(\omega_{m}\right)}{\sqrt{\left(\omega_{m}+\Sigma_{T}\left(\omega_{m}\right)\right)^{2}+\tilde{v}_{F}^{2}|\mathbf{p}|^{2}}} \frac{f^{2}\left(\theta_{k}\right)}{M^{2}+|\mathbf{p}|^{2}} \\
& \quad=\frac{\bar{g} T f^{2}\left(\theta_{k}\right) \sigma\left(\omega_{m}\right)}{2 \pi\left|\omega_{m}+\Sigma_{T}\left(\omega_{m}\right)\right|} \mathcal{S}\left(\frac{v_{F} M}{\left|\omega_{m}+\Sigma_{T}\left(\omega_{m}\right)\right|}\right),
\end{aligned}
$$

where

$$
\mathcal{S}(x)=\frac{\cosh ^{-1}(1 / x)}{\sqrt{1-x^{2}}} \approx \begin{cases}\log (2 / x) & x \ll 1 \\ \pi /(2 x) & x \gg 1\end{cases}
$$

and $\tilde{v}_{F}=v_{F}\left(\theta_{k+Q}\right)$, i.e., $\tilde{v}_{F}=\left|v_{F}\left(\theta_{h s}\right)\right|$ for the spinfermion model and $\tilde{v}_{F}=v_{F}\left(\theta_{k}\right)$ for the Ising-nematic model. Recall that we set $f(\theta)=1$ for the spin-fermion model. For the Ising-nematic model, the typical momentum transfers along the FS are small, which explains the presence of $f^{2}\left(\theta_{k}\right)$ in the rhs of Eq. (25).

We assume that close enough to the QCP, $v_{F} M \ll$ $\left|\omega_{m}\right|+\left|\Sigma_{T}\left(\omega_{m}\right)\right|$ for all Matsubara numbers (this will allow us to obtain analytic expressions in what follows). Using the form of $S(x)$ in this limit, we can solve Eq. (25) to obtain an analytic expression for $\Sigma_{T}\left(\omega_{m}\right)$. For $\omega_{m}>0$,

$$
\begin{aligned}
\Sigma_{T}\left(\omega_{m}, \theta_{k}\right) \\
\quad \approx \sqrt{\omega_{T}\left(\theta_{k}\right)^{2} \log \frac{\sqrt{4 \omega_{T}\left(\theta_{k}\right)^{2}+\omega_{m}^{2}}+\omega_{m}}{v_{F} M}+\frac{\omega_{m}^{2}}{4}-\frac{\omega_{m}}{2}} \\
\quad= \begin{cases}\omega_{T}\left(\theta_{k}\right)-\frac{\omega_{m}}{2} & \omega_{m} \ll \omega_{T}\left(\theta_{k}\right) \\
\frac{\omega_{T}\left(\theta_{k}\right)^{2}}{\omega_{m}} & \omega_{m} \gg \omega_{T}\left(\theta_{k}\right),\end{cases}
\end{aligned}
$$

where

$$
\begin{aligned}
\omega_{T}\left(\theta_{k}\right) & =\omega_{T}^{0}\left|f\left(\theta_{k}\right)\right| \sqrt{\log \frac{2 \omega_{T}^{0}\left|f\left(\theta_{k}\right)\right|}{v_{F} M}}, \\
\omega_{T}^{0} & =\sqrt{\frac{N_{b} \bar{g} T}{2 \pi}}
\end{aligned}
$$

and we neglected $\log (\log (\cdots))$ terms. We show the result in Fig. 3. This expression was also obtained in Ref. [57]. It is worth mentioning that $\omega_{T}$ from Eq. (28) has almost the same temperature dependence as is found when $\Sigma_{T}$ is evaluated with factorization of momentum since, in that case, $\Sigma_{T} \sim \bar{g} T / v f M(T) \sim \sqrt{T}$ if we assume Curie-Weiss behavior for $M(T)$, as was done in, e.g., Ref. [49] (up to logarithmic factors). However, as discussed above, the physical mechanism is different.

The crossover between the two asymptotic behaviors of $\Sigma_{T}$ occurs at $\omega_{m} \sim \omega_{T}$, i.e., at a typical Matsubara number

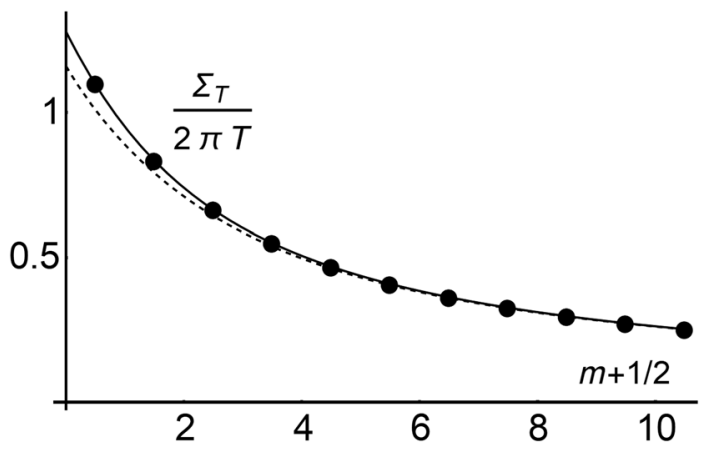

FIG. 3. The thermal contribution $\Sigma_{T}\left(\omega_{m}\right)$ to the fermionic selfenergy. The black dots are a numerical solution of Eq. (25). The dashed line is the analytic approximation in Eq. (27), and the solid black line is a solution with higher accuracy, up to $\log \log \log (\cdots)$ terms. The numerical solution was obtained for $\left(2 \pi T / v_{F} M\right)=(\bar{g} / 2 \pi T)=10$.

$$
m_{T} \equiv \frac{\omega_{T}}{T} \sim \sqrt{\frac{\bar{g}}{T}} \sqrt{\log \frac{\sqrt{\bar{g} T}}{v_{F} M}}
$$

Next, we determine the dynamic contribution $\Sigma_{Q}$-the sum over nonzero bosonic frequencies in Eq. (18). As we discussed earlier, we assume that this contribution does not require self-consistency and that it is sufficient to replace $\Sigma\left(\omega_{m}\right)$ by $\Sigma_{T}$ in the rhs of Eq. (18). For the spin-fermion model, we find

$\Sigma_{Q}^{\mathrm{SFM}}\left(\omega_{m}, \theta_{k}=\theta_{h s}\right)$

$$
\approx \frac{N_{b} \bar{g} T}{2 \pi} \sum_{n \neq m} \frac{\sigma\left(\omega_{n}\right)}{\left|\omega_{n}+\Sigma_{T}\left(\omega_{n}\right)\right|} \mathcal{S}\left(\frac{v_{F} \sqrt{M^{2}+\Pi^{\mathrm{SFM}}\left(\omega_{m}-\omega_{n}\right)}}{\left|\omega_{n}+\Sigma_{T}\left(\omega_{n}\right)\right|}\right),
$$

where $\mathcal{S}(x)$ is the same as in Eq. (26), but the argument now contains the polarization bubble $\Pi^{\mathrm{SFM}}$ next to $M^{2}$. This addition of $\Pi^{\mathrm{SFM}}$ implies that the crossover between two limiting forms of $\mathcal{S}(x)$ now holds even if $M=0$; i.e., there are two different behaviors of $\Sigma_{Q}^{\mathrm{SFM}}\left(\omega_{m}, \theta_{k}\right)$ near a QCP. To find out at what $m$ the crossover occurs, we need to know $\Pi^{\mathrm{SFM}}\left(\omega_{m}-\omega_{n}, Q_{h s}\right)$ at a finite temperature. Examining Eq. (17) for $\Pi\left(\Omega_{m}, Q_{h s}\right)$, we find that corrections to the zero-temperature form, Eq. (20), are suppressed by powers of $T / E_{F}$ and are therefore irrelevant. Accordingly, we simply plug the $T=0$ result into Eq. (30). We assume and then verify that the argument of $\mathcal{S}$ in Eq. (30) is of order one at $m<m_{T}$, where $\omega_{m}<\Sigma_{T}\left(\omega_{m}\right)$. Neglecting $\omega_{m}$ compared to $\Sigma_{T}\left(\omega_{m}\right)$, using the fact that typical internal $n$ are comparable to $m$ and using $\Sigma_{T}\left(\omega_{m}\right) \sim \omega_{T} \sim \sqrt{\bar{g} T \log \left(\sqrt{\bar{g}} T / v_{F} M\right)}$, $\sqrt{\Pi\left(\omega_{m}-\omega_{n}, Q_{h s}\right)} \sim \sqrt{\nu_{F} \bar{g} \omega_{m} / v_{F} Q_{h s}}$, and $\nu_{F} v_{F} / Q_{h s}=$ $O(1)$, we find that the crossover in $\Sigma_{Q}^{\mathrm{SFM}}\left(\omega_{m}, \theta_{h s}\right)$ occurs at $\omega_{m} \sim \omega_{T}^{\prime} \sim \omega_{T}(T / \bar{g})^{1 / 2} \ll \omega_{T}$, i.e., at a typical Matsubara number 


$$
m_{T}^{\prime} \sim \log \frac{\sqrt{\bar{g} T}}{v_{F} M}
$$

Clearly, $m_{T}^{\prime} \ll m_{T}$ at low enough temperatures. This case justifies the use of $m<m_{T}$ in the derivation of Eq. (31). The two limiting forms of $\Sigma_{Q}^{\mathrm{SFM}}\left(\omega_{m}, \theta_{h s}\right)$ are

$$
\Sigma_{Q}^{\mathrm{SFM}}\left(\omega_{m}, \theta_{h s}\right) \approx \frac{\bar{g} \omega_{m}}{2 \pi^{2} \omega_{T}\left(\theta_{h s}\right)} L\left(m, \omega_{m}\right)
$$

at $\omega_{m} \ll \omega_{T}^{\prime}$, where

$$
L \approx \log \left(\frac{\omega_{T}}{v_{F} \sqrt{\bar{g}\left|\omega_{m}\right|}}\right)
$$

and

$$
\Sigma_{Q}^{\mathrm{SFM}}\left(\omega_{m}, \theta_{h s}\right) \approx \omega_{\mathrm{SFM}}^{1 / 2} \omega_{m}^{1 / 2}
$$

for $\omega_{m} \gg \omega_{T}^{\prime}$. This form is the same as at $T=0$.

We now combine our results for the thermal and quantum parts of the self-energy and show that there are two asymptotic behaviors for $\Sigma\left(\omega_{m}\right)$ separated by a wide regime.

Region I: The strongly thermal regime.-The strongly thermal regime occurs at $\omega_{m} \ll \omega_{T}^{\prime} \ll \omega_{T}$, i.e., $m \ll m_{T}^{\prime} \ll$ $m_{T}$. In this case, adding up the appropriate limits from Eqs. (27) and (32), we find that the self-energy has the form

$$
\Sigma^{\mathrm{SFM}}\left(\omega_{m}\right) \approx \omega_{T}\left(1+A \frac{\omega_{m}}{\omega_{T}}\right),
$$

where the leading term comes from the frequency-independent part of $\Sigma_{T}$, and the subleading term, with prefactor $A$, is the combination of a frequency-dependent term in $\Sigma_{T}$ and from $\Sigma_{Q}$, in Eqs. (27) and (32). At vanishing $M, A \approx-1 / 2$, and the full $\Sigma\left(\omega_{m}\right)$ comes from $\Sigma_{T}$. However, the $O\left(\omega_{m}\right)$ term from $\Sigma_{Q}$ is only logarithmically reduced and, in practice, contributes nearly equally to $A$. In this regime, there is no factorization of momentum even for $\Sigma_{Q}$, and ET is qualitatively invalid.

Region II: The almost-critical regime.-The almostcritical regime occurs for $\omega_{m} \gg \omega_{T} \gg \omega_{T}^{\prime}$, i.e., $m \gg m_{T} \gg$ $m_{T}^{\prime}$. Here, summing up the appropriate expressions in Eqs. (27) and (34), we find that the total fermionic selfenergy has the form

$$
\Sigma^{\mathrm{SFM}}\left(\omega_{m}\right) \approx \omega_{\mathrm{SFM}}^{1 / 2} \omega_{m}^{1 / 2}+\frac{\omega_{T}^{2}}{\omega_{m}} .
$$

The first term (the same one as at $T=0$ ) comes from $\Sigma_{Q}$; the second one comes from $\Sigma_{T}$. Because $\omega_{\mathrm{SFM}} \sim \bar{g}$, the first term is the dominant one for $m>m_{T}$; i.e., the self-energy is predominantly determined by dynamical quantum fluctuations. In this regime, $\Sigma_{Q}$ is identical to the ET result. A similar separation into thermal and critical regions was found in Ref. [52].
Note that because $\Sigma_{Q}\left(\omega_{m}\right)$ changes its behavior at $\omega_{m} \sim \omega_{T}^{\prime}$ and $\Sigma_{T}\left(\omega_{m}\right)$ changes its behavior at $\omega_{m} \sim \omega_{T}$, there is no single crossover from the region where thermal self-energy dominates to the one where the dynamical selfenergy dominates. In between the two limiting regimes $\omega_{m} \ll \omega_{T}^{\prime}$ and $\omega_{m} \gg \omega_{T}$, there is a wide intermediate region of $\omega_{T}^{\prime} \ll \omega \ll \omega_{T}\left(m_{T}^{\prime} \ll m \ll m_{T}\right)$, where quantum and thermal contributions are comparable. We stated this result in the Introduction, Eq. (5). The implication of Eqs. (35) and (36) is that the behavior of $\Sigma$ at the critical point is quite involved. While at high frequencies the behavior will tend to a power law, at lower frequencies the self-energy saturates and appears to reach a plateau.

Before moving on to the Ising-nematic model, we need to go back and check whether we were justified in neglecting the contribution of $\Sigma_{Q}$ in comparison with $\Sigma_{T}$ in the self-consistent calculation of $\Sigma_{T}$ and the subsequent calculation of $\Sigma_{Q}$. From Eqs. (35) and (36), it is evident that, in the strongly thermal region, $\Sigma_{Q} \ll \Sigma_{T}$ by a logarithmic factor, while in the almost critical regime, $\Sigma_{T} \ll \omega_{m}$; we already know from ET (see Sec. II C) that the contribution of $\Sigma_{Q}$ can be neglected when computing $\Sigma_{Q}$. In the intermediate regime, the approximation is not rigorously justified since both $\Sigma_{T}$ and $\Sigma_{Q}$ scale as $\sqrt{\bar{g}} T$ and both are larger than $\omega_{m}$, but it serves as a way to interpolate between the two regimes.

For the Ising-nematic model, the behavior is somewhat more complex due to the momentum dependence of the polarization. The thermal contribution $\Sigma_{T}\left(\omega_{m}\right)$ is identical to that for the SFM and is given by Eq. (27). The dynamical contribution is, from Eq. (23),

$$
\begin{aligned}
\Sigma_{Q}^{\mathrm{INM}}\left(\omega_{m}\right) \approx & \frac{\bar{g} T f^{2}\left(\theta_{k}\right)}{2 \pi} \sum_{n \neq m} \frac{\sigma\left(\omega_{n}\right)}{\left|\omega_{n}+\Sigma_{T}\left(\omega_{n}\right)\right|} \\
& \times \mathcal{T}\left(\frac{v_{F} M}{p_{n}}, \frac{\left(\Pi^{\mathrm{INM}}\left(\omega_{n}-\omega_{m}, p_{n}\right)\right)^{1 / 3}}{p_{n}^{2 / 3}}\right),
\end{aligned}
$$

where $v_{F} p_{n}=\left|\omega_{n}+\Sigma_{T}\left(\omega_{n}\right)\right|, \Pi^{\mathrm{INM}}\left(\Omega_{m}, q\right)$ is given by Eq. (21), and

$$
\begin{aligned}
\mathcal{T}(x, y) & =\int_{0}^{\infty} \frac{z^{2} d z}{\sqrt{z^{2}+1}\left(z^{3}+z x^{2}+y^{3}\right)} \\
& = \begin{cases}\log (2 / x) & y=0, x \rightarrow 0 \\
\log (2 / y) & x=0, y \rightarrow 0 \\
2 \pi /(3 \sqrt{3} y) & x=0, y \gg 1\end{cases}
\end{aligned}
$$

An analysis similar to the one we performed for the spin-fermion model shows that at $M=0$, $\Sigma_{Q}^{\mathrm{INM}}\left(\omega_{m}\right)$ undergoes a crossover at $\omega_{T}^{\prime} \sim \omega_{T}\left(\omega_{T}^{2} /\left(\bar{g} E_{F}\right)\right) \sim$ $\omega_{T}\left(T \log \left(\sqrt{\bar{g}} T / v_{F} M\right) / E_{F}\right) \quad$ between $\quad \Sigma_{Q}^{\mathrm{INM}}\left(\omega_{m}\right) \sim$ $\left(\omega_{m} / \omega_{T}\right) \log \left(\nu_{F} \bar{g} \omega_{m} v_{F}^{2} / \omega_{T}^{3}\right)$ at $\omega_{m}<\omega_{T}^{\prime}$ and $\Sigma_{Q}^{\mathrm{INM}}\left(\omega_{m}\right) \sim$ $\omega_{m}^{2 / 3}\left(\omega_{\text {INM }}\right)^{1 / 3}$ at $\omega_{m}>\omega_{T}^{\prime}$. The crossover frequency $\omega_{T}^{\prime}$ is 
different from the one for the spin-fermion model but also obeys the condition $\omega_{T}^{\prime} \ll \omega_{T}$. Comparing the results for $\Sigma_{Q}^{\mathrm{INM}}\left(\omega_{m}\right)$ and $\Sigma_{T}\left(\omega_{m}\right)$, Eq. (26), we obtain the limiting forms of $\Sigma^{\mathrm{INM}}$ in region I, $\omega_{m}<\omega_{T}^{\prime}$, and region II, $\omega_{m}>\omega_{T}$ :

$$
\Sigma\left(\omega_{m}\right) \approx \begin{cases}\omega_{T}\left(1+B \frac{\omega_{m}}{\omega_{T}}\right) & \text { region I } \\ \omega_{\mathrm{INM}}^{1 / 3} \omega_{m}^{2 / 3}+\frac{\omega_{T}^{2}}{\omega_{m}} & \text { region II, }\end{cases}
$$

where $B$ is a constant. (Note that since $\omega_{T}^{\prime} / \omega_{T} \sim \omega_{T}^{2} / \bar{g} E_{F}$, for low enough temperatures, $\omega_{T}^{\prime}<T$, and region $\mathrm{I}$ is inaccessible.)

The bosonic self-energy.-We now discuss the bosonic self-energy in more detail. From Eq. (17), we see that corrections to the $T=0$ form of $\Pi$ comes from the term $\omega_{m}+\Sigma\left(\omega_{m}\right)$, which means that the behavior of $\Pi$ depends on whether we are in region I or II. In region II, we may neglect self-energy corrections to $\Pi$, and it will retain its $T=0$ form. In region I, there is a different behavior for the spin-fermion model vs the Ising-nematic model. In the spin-fermion model, the typical momentum transfer is of order $v_{F} Q_{h s} \sim E_{F}$; hence, $v_{F} Q_{h s} \gg \omega_{T}$ even at finite $T$, and the $T=0$ result holds. In the Ising-nematic model, the typical momentum transfer is $v_{F}|\mathbf{q}| \sim\left(\bar{g} E_{F} T\right)^{1 / 3} \gg \omega_{T}$, so the $T=0$ result can be used in evaluating the fermionic self-energy. However, since QMC simulations also measure $\Pi\left(\Omega_{m},|\mathbf{q}|\right)$ for a given external $\Omega_{m},|\mathbf{q}|$, and since there is a large parameter range where $\left|\omega_{m}\right| \ll v_{F}|\mathbf{q}| \ll \omega_{T}$, we need to calculate $\Pi$ by taking into account the self-energy contribution.

For the computation $\Pi$ in the Ising-nematic model, it is important to realize that a nematic-order parameter is not a conserved quantity, such as, e.g., a spin-order parameter (the total magnetization) in a ferromagnet. For a conserved order parameter, a Ward identity ensures that, within ET, vertex corrections cancel out exactly so that the one-loop calculation that is analogous to Eq. (21) is exact [58]. In the Ising-nematic model, this cancellation does not occur; hence, at low frequencies, self-energy corrections become important. This is true even in the $T=0$ limit [51].

To gain a qualitative understanding of the impact of the self-energy, we evaluate Eq. (17), keeping only the thermal part of the fermionic self-energy. To leading order in $\omega$, we can treat the self-energy as a constant $\Sigma_{T}\left(\omega_{n}, \theta_{k}\right) \approx$ $\omega_{T}\left(\theta_{k}\right) \sigma\left(\omega_{n}\right)$. We obtain

$$
\begin{aligned}
\Pi^{\mathrm{in}}\left(\Omega_{m}, \mathbf{q}\right) & \approx 2 i \bar{g} T \sum_{n} \int \frac{d \theta}{2 \pi} \nu_{F}(\theta) f^{2}(\theta) \frac{\Theta\left(\omega_{n+m}\right)-\Theta\left(\omega_{n}\right)}{i \omega_{T}(\theta)\left(\sigma\left(\omega_{n+m}\right)-\sigma\left(\omega_{n}\right)\right)-v_{F}(\theta) q \cos \left(\theta-\theta_{q}\right)} \\
& \approx \frac{\bar{g}}{2 \pi^{2}}\left|\Omega_{m}\right| \int d \theta \nu_{F}(\theta) f^{2}(\theta) \frac{2\left|\omega_{T}(\theta)\right|}{4 \omega_{T}^{2}(\theta)+v_{F}^{2}(\theta) q^{2} \cos ^{2}\left(\theta-\theta_{q}\right)} \\
& \approx \bar{g} \frac{\tilde{\nu}_{F}}{2 \pi} \frac{\left|\Omega_{m}\right|}{\omega_{T}(\theta=0)},
\end{aligned}
$$

where $\tilde{\nu}_{F}=(2 \pi)^{-1} \int \nu_{F}(\theta)|f(\theta)|$, and the last line is valid for $q \rightarrow 0$. The detailed behavior of $\Pi$ as a function of $\Omega_{m}, \Sigma\left(\omega_{m}\right), q$, and $\theta$ is more complicated and is obtained by a numerical integration of Eq. (40).

Equations (37) and (41) for the Ising-nematic model and Eqs. (20) and (32) for the spin-fermion model show remarkably similar behavior. The fermionic response is the same for both systems, up to some model-dependent constants. The bosonic response is featureless and linear in $\Omega_{m}$, which implies that the fermionic self-energy will be qualitatively the same as for the spin-fermion model in both models.

\section{LATTICE THEORY}

Before beginning our analysis of the QMC data, we first briefly describe the lattice version of the self-consistent equations (14) and (15), which we refer to as the lattice theory (LT). As we discussed, comparing ET, LT, and QMC results gives us insight into the role of high-energy fermions in contributing to the self-energy.

The spin-fermion and Ising-nematic models are defined on a finite space-time lattice of $L \times L \times L_{\tau}$ sites with periodic boundary conditions in the spatial directions and periodic (antiperiodic) boundary conditions for the bosons (fermions) in the imaginary time direction. For both models, the bosonic part of the action is given by the lattice version of Eq. (8), with an additional dynamical term

$\mathcal{S}_{b}=D_{0}^{-1} \int d \tau \sum_{\mathbf{q}} \phi(\mathbf{q})\left(M_{0}^{2}-\partial_{x}^{2}-\partial_{y}^{2}-c^{-2} \partial_{\tau}^{2}\right) \phi(\mathbf{q})$.

Here, $\partial_{x, y, \tau}$ is understood as a discretized derivative, and $c$ is the bare velocity of the boson. We introduce this additional dynamical term to better match the QMC lattice 
models, where the bosonic fields have their own independent dynamics.

In the Ising-nematic case, the form factor is slightly modified compared to the definition used in Sec. II A,

$$
\mathcal{S}_{\mathrm{I}}=\frac{g}{L} \int d \tau \sum_{\mathbf{k} \mathbf{q} \sigma} \psi_{\sigma}^{\dagger}(\mathbf{k}-\mathbf{q}) \psi_{\sigma}(\mathbf{k}) \phi(\mathbf{q}) f(\mathbf{k}, \mathbf{q})
$$

where $f(\mathbf{k}, \mathbf{q})=\cos \left(q_{x} / 2\right) \cos \left(k_{x}-q_{x} / 2\right)-\cos \left(q_{y} / 2\right) \times$ $\cos \left(k_{y}-q_{y} / 2\right)=\cos k_{x}-\cos k_{y}+O(q)$.

The self-energies then take the form

$$
\begin{aligned}
& i \Sigma(k)=\frac{g^{2} T}{L^{2}} \sum_{q} f^{2}(\mathbf{k}, \mathbf{q}) D(q) G(k+q), \\
& \Pi(q)=\frac{2 g^{2} T}{L^{2}} \sum_{k} f^{2}(\mathbf{k}, \mathbf{q}) G(k) G(k+q) .
\end{aligned}
$$

The fermionic part of the action is given by Eq. (7), where $\epsilon_{\mathbf{k}}$ is a nearest-neighbor tight-binding dispersion.

In the spin-fermion case, we consider the two-band model depicted in Fig. 4. Again, reason for this modification from the model depicted in Fig. 1(a) is to better match the sign problem-free model that was simulated in QMC. The fermionic part of the action is

$$
\mathcal{S}_{f}=\int d \tau \sum_{\mathbf{k} \eta \sigma} \psi_{\eta \sigma}^{\dagger}(\mathbf{k})\left(\partial_{\tau}-\epsilon_{\eta, \mathbf{k}}\right) \psi_{\eta \sigma}(\mathbf{k})
$$

where $\eta= \pm 1$ is the band index. The interaction part of the action is

$$
\mathcal{S}_{I}=\frac{g}{L} \int d \tau \sum_{\mathbf{k} \mathbf{q} \sigma \sigma^{\prime} \eta} \vec{\phi}(\mathbf{Q}+\mathbf{q}) \psi_{\eta \sigma}^{\dagger}(\mathbf{k}) \vec{\tau}_{\sigma \sigma^{\prime}} \psi_{-\eta \sigma^{\prime}}(\mathbf{k}+\mathbf{Q}+\mathbf{q})
$$

Finally, the LT self-energies are given by

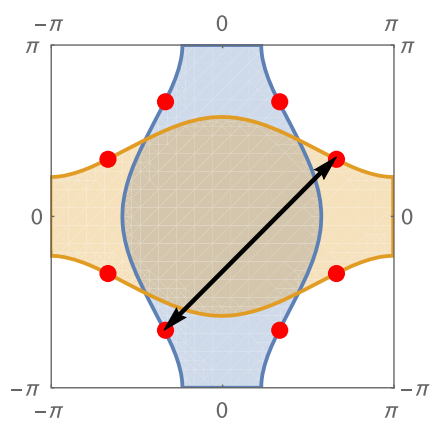

FIG. 4. FS structure of the two-band spin-fermion model used in the QMC simulations and the LT calculations. Hotspots are indicated in red and are connected by the $\mathrm{AF}$ wave vector $\mathbf{Q}=(\pi, \pi)$, denoted by an arrow.

$$
\begin{aligned}
i \Sigma_{\eta}(k) & =\frac{g^{2} N_{b} T}{L^{2}} \sum_{q} D(q) G_{-\eta}(k+q), \\
\Pi(q) & =\frac{2 g^{2} T}{L^{2}} \sum_{k \eta} G_{\eta}(k) G_{-\eta}(k+q) .
\end{aligned}
$$

We solve the LT equations by using the iteration method. The local nature of the boson-fermion coupling allows us to rewrite the Eliashberg equations in real space and imaginary time. We then evaluate the self-energies by performing a fast Fourier transform, with a computational cost of $L^{2} L_{\tau} \log \left(L^{2} L_{\tau}\right)$ per iteration. Note that a naive Fourier transform of the fermionic Green's function to Matsubara frequencies generates an error that scales as $O\left(1 / T L_{\tau}\right)$. For better convergence, we use the "Filon-trapezoidal" rule [59] to reduce the error to $O\left(1 / T L_{\tau}\right)^{2}$.

\section{COMPARISON TO LATTICE THEORY AND TO QUANTUM MONTE CARLO SIMULATIONS}

In this section, we compare our finite-temperature results for the self-energy with QMC results for the spin-fermion and Ising nematic models. We compare QMC results to the expressions for $\Sigma(k)$ and $\Pi(q)$, which we obtained within MET as well as within LT. We see that the functional forms of $\Sigma(k)$ and $\Pi(q)$ obtained within MET and LT are similar but with some differences. By comparing these three forms of $\Sigma$ and $\Pi$, we are able to both verify the validity of the MET and identify the strength of vertex corrections, which are not included in MET and LT, and lattice effects, which are not included in MET but are present in LT.

Before going into a detailed comparison, we discuss the relationship between MET, LT, and QMC calculations and show how to compare them.

The models used in the QMC studies differ from the ones we introduced in Sec. II in several ways. First, in the QMC models, the bosonic degrees of freedom have their own dynamics. Nevertheless, at low frequencies, relevant to the physics near the QCP, the leading term in the bosonic dynamics is the Landau damping due to coupling to electronic degrees of freedom since the Landau damping term scales as $|\Omega|$ while the dynamical term of a boson scales as $\Omega^{2}$. Second, the models used in the QMC studies contain additional interactions, which are not present in the MET or LT. These interactions include boson-boson interactions, as well as (in the Ising-nematic model) additional, noncritical bosonic modes that couple to the fermions. Finally, the three methods differ in the proper definition of the parameters of $D_{0}, M(T)$, and $g$. The QMC starts from the theory with bare parameters and returns the numerically exact $G(k), D(q)$, so that $D_{0}, M(T)$ are outputs of the calculation. In the MET and LT, $D_{0}, M(T)$ are considered as inputs to the theory. The bare parameter values are renormalized by vertex corrections and, in the case of MET, 
one-loop self-energy corrections from high-energy fermions. These renormalizations should be absorbed into the input of the theory, which implies that the effective $g$ may be different in MET and LT. We obtain $D_{0}$ and $M(T)$ by fitting the static $D\left(q, \Omega_{m}=0\right)$ from QMC, and we use the bare value of $g$, as elaborated below. We find that the bare $g$ reproduces the QMC data well for both the spinfermion and Ising-nematic models.

We first show our results for the spin-fermion model. References $[39,40,48]$ presented extensive QMC data on a realization of the spin-fermion model. To compare with our work, we use the data from those papers for $g=1.5, T=0.05, \ldots, 0.2$, where all energies are in units of the bare hopping used in Refs. [39,40,48]. We note that for the lowest temperature, $T=0.05$, the thermal scale, given by Eq. (28), $\omega_{T}^{0}=0.16$, is almost the same as the lowest Matsubara frequency $\pi T$, and in addition, $v_{F} M(T) \gtrsim \pi T, \omega_{T}^{0}$. The implication is that the QMC data mostly fall into the intermediate regime between regions I and II, and the system is not fully critical. For this reason, for the quantitative comparison of the low-energy MET with QMC, we performed the Matsubara summations in Eq. (30) numerically.

We begin by comparing the self-energies $\Sigma_{T}\left(\omega_{m}\right)$ and $\Sigma_{Q}\left(\omega_{m}\right)$ within MET and LT. Figure 5 presents $\Sigma_{T}, \Sigma_{Q}$, and $\Sigma$ at $\theta=\theta_{h s}$ for both methods. Although there are some deviations, the overall results are similar. We also note the somewhat curious feature, shown in Figs. 5(b) and 5(e), that for both theories, the quantum self-energy at the first Matsubara frequency $\Sigma_{Q}(\pi T)$ is negative [it is also true for the Ising-nematic model, Figs. 8(b) and 8(e)]. A similar property of the real part of the self-energy in real frequencies was found in Ref. [49]. We provide a transparent derivation of this property of $\Sigma_{Q}(\pi T)$ in the Appendix.

In Fig. 6, we compare the QMC data with both calculations. Except for a global discrepancy of about $20 \%$ for the MET, and a slight difference in slope for the LT, there is good agreement with QMC. The overall magnitude agrees well, and both the MET and the LT reproduce the QMC result that $\Sigma$ seems to extrapolate to a

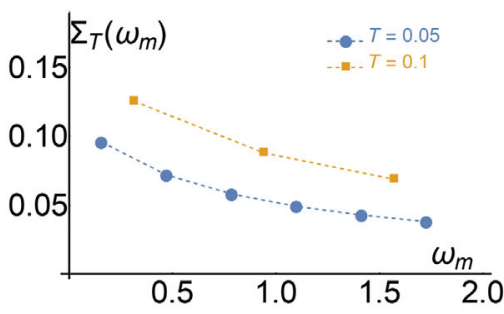

(a)

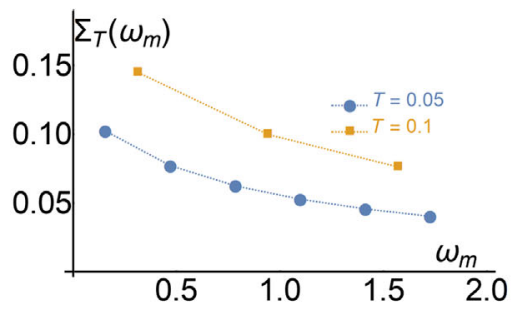

(d)

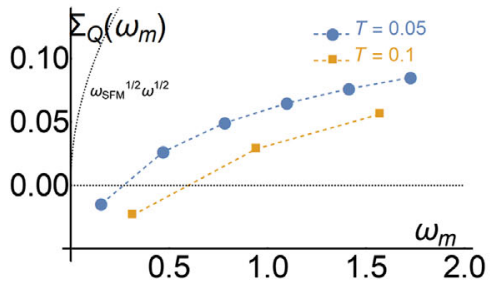

(b)

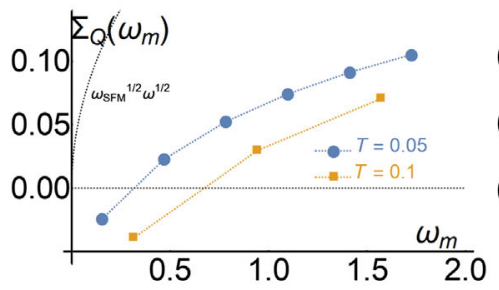

(e)

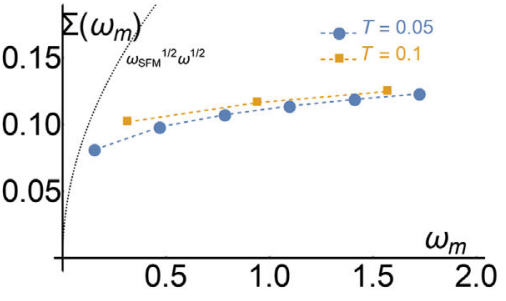

(c)

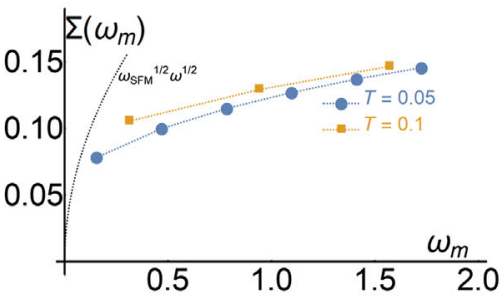

(f)

FIG. 5. Fermionic self-energy for the spin-fermion model. (a)-(c) Dashed traces: Fermionic self-energy from MET, Eq. (24), showing the (a) thermal, (b) quantum, and (c) total self-energy. The black dashed line on the right is the asymptotic $T=0$ prediction of ET. (d)-(f) Dotted traces: Fermionic self-energy from LT.

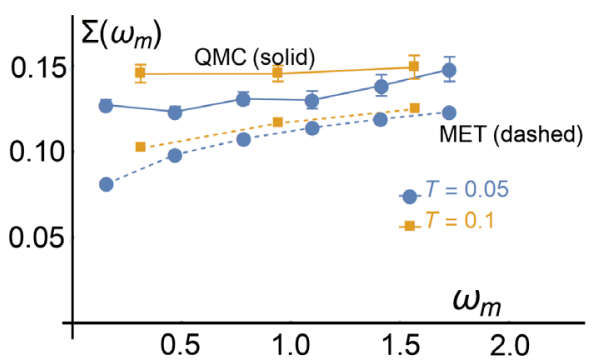

(a)

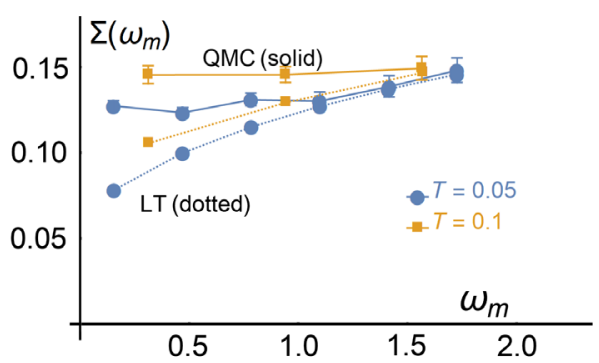

(b)

FIG. 6. Fermionic self-energies in the spin-fermion model. (a) Comparison of the MET and QMC self-energy. (b) Comparison of the LT and QMC self-energy. 


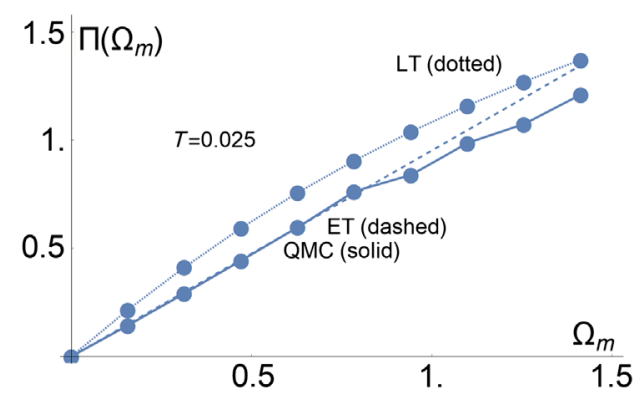

FIG. 7. Bosonic self-energy $\Pi\left(\Omega_{m}\right)$ in the spin-fermion model for $q=0$. In this figure, the dashed trace corresponds to the $T=0$ (ET) expression, Eq. (20). Error bars on the QMC data in this and subsequent figures are smaller than the data point sizes.

nonzero value as $\omega_{m} \rightarrow 0$. We note that the overall good agreement between MET, LT, and QMC calculations holds despite the fact that the coupling constant $\bar{g} \approx 1.67$ is not small compared to $E_{F} \approx 2.2$, implying, e.g., that vertex corrections should generally be $O(1)$. In Figs. 5 and 6, we show only two of the temperatures so as to keep the images clear, but the same agreement holds for the other temperatures. For completeness, we also display a comparison of QMC, MET, and LT for $\Pi\left(\Omega_{m}\right)$ in Fig. 7, showing good agreement. As discussed at the end of Sec. III, for the spinfermion model, $\Pi\left(\Omega_{m}\right)$ is expected to show good agreement with the $T=0$ ET even at finite temperatures because typical momentum transfer $v_{F} Q_{h s}$ is large.

Next, we consider the Ising-nematic model. While in the spin-fermion case, the model used in QMC allows us to directly read off the bare value for the coupling constant $g$, the nematic modes in the QMC studies $[11,41,48]$ consist of pseudospin- $1 / 2$ degrees of freedom that are located on the bonds of the lattice and do not directly match the form of Eqs. (11) and (43). Nevertheless, the bare $g$ can be obtained by comparing the interaction term for a uniform $(\mathbf{q}=0)$ configuration of the nematic modes. This process yields $g=3.3 \alpha$ for MET and $g=2 \alpha$ for the LT, where $\alpha$ is the coupling to the pseudospin degrees of freedom in the notation of Refs. [11,41,48].

Here, we focus on the data for $\alpha=1$ and $T=0.1,0.167$, 0.25 [60]. Extracting $D_{0}$ from the QMC data, we find that the bare coupling used in MET is $\bar{g}=g^{2} D_{0}=8.25$. We note in passing that $\mathrm{QMC}$ simulations show a $T_{c}=0.04$, which is in good agreement with the ET prediction of $T_{c, \mathrm{ET}}=0.066$ for the given $\bar{g}$. The Eliashberg $T_{c}$ in the Ising-nematic model scales as $\bar{g}^{2} / E_{F} \propto g^{4}$, so the difference in critical temperatures can be interpreted as a $15 \%$ renormalization of $g$. In the LT, we find $T_{c, \mathrm{LT}}=0.03$. Figure 8 presents $\Sigma_{T}, \Sigma_{Q}$ and $\Sigma$ at $\theta=0$ for both methods.

Figure 9 presents a comparison of the QMC data for $\Sigma$ with both the MET and LT calculations. The MET calculation shows an excellent agreement with the data. The LT calculation has some systematic deviations in the frequency behavior. From all of these results, we conclude that, similarly to the spin-fermion model, there are only moderate high-energy vertex corrections and lattice renormalizations in the Ising-nematic model, and they are somewhat better accounted for in the low-energy MET.

We also compared MET and lattice calculations to the QMC data for $\Pi(q)$. In Fig. 10, we present the behavior of $\Pi$ at the lowest $q=2 \pi / L$, where $L$ is the QMC system

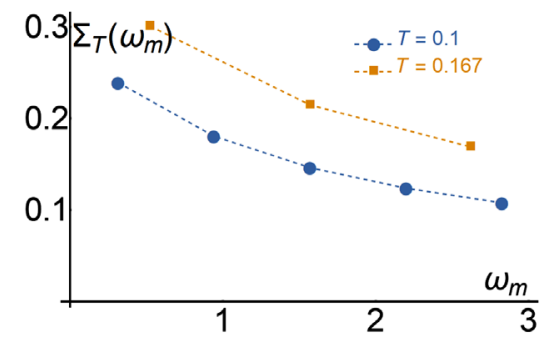

(a)

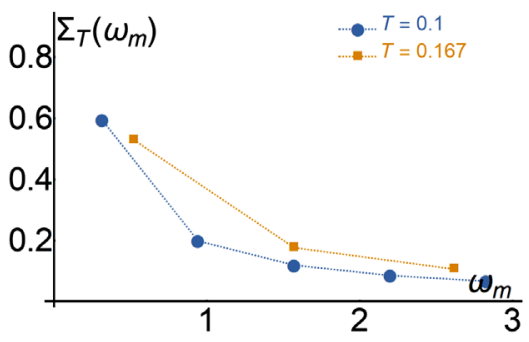

(d)

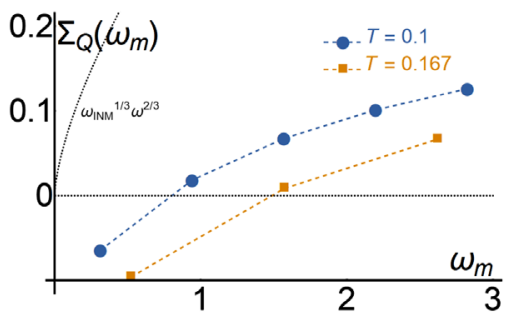

(b)

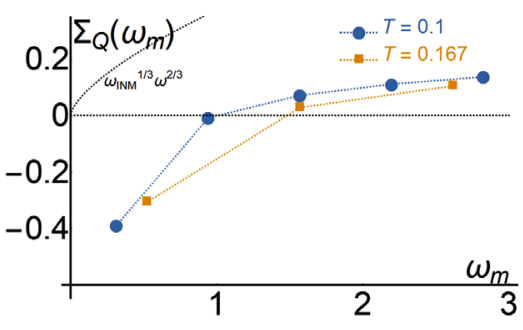

(e)

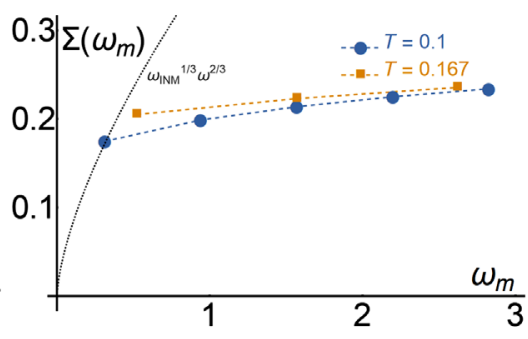

(c)

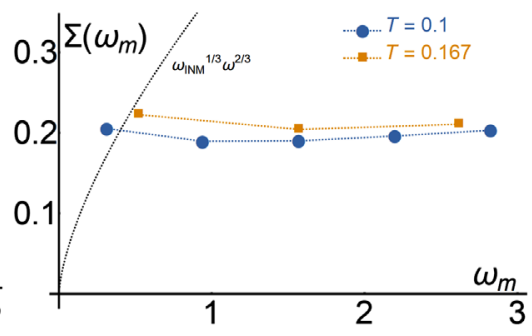

(f)

FIG. 8. The fermionic self-energy for the Ising-nematic model. (a)-(c) Dashed traces: Fermionic self-energy from MET, showing the (a) thermal, (b) quantum, and (c) total self-energy. The black dashed lines on the right are the asymptotic $T=0$ prediction of ET. (d)-(f) Dotted traces: Fermionic self-energy from LT. 


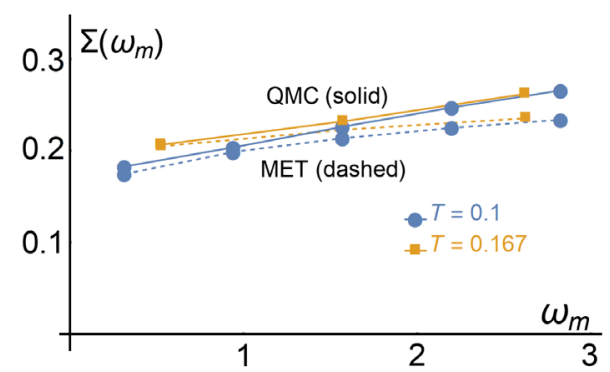

(a)

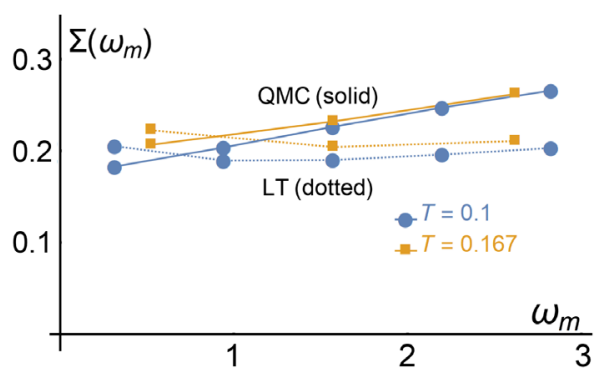

(b)

FIG. 9. Fermionic self-energy of the Ising-nematic model. (a) Comparison of the MET and QMC self-energy. (b) Comparison of the LT and QMC self-energy.

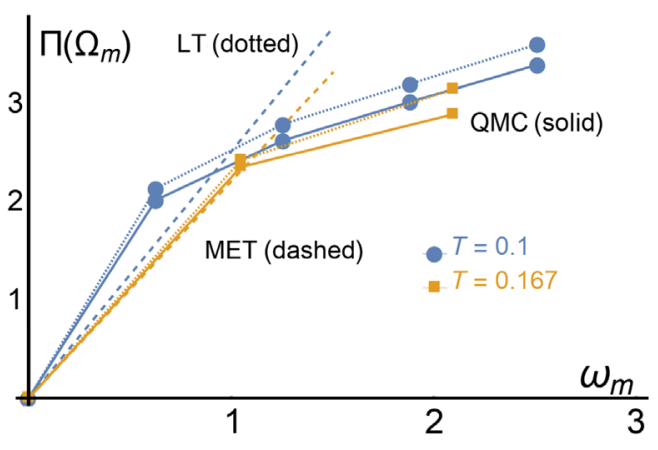

FIG. 10. The bosonic self-energy $\Pi\left(\Omega_{m}\right)$ in the Ising-nematic model for the lowest $q=2 \pi / L$, where $L$ is the system size. Solid lines denote the QMC data, and dashed and dotted lines denote the MET and LT calculations.

size, along with a lattice calculation of $\Pi$ and the MET prediction of the low-frequency behavior of $\Pi$, Eq. (41) [61]. There is good agreement between MET/LT and the QMC data.

We find it striking that most of the features of the QMC data are reproduced by our ET/MET calculations. We believe that our results imply that the QMC data are a validation of, rather than a challenge to, the applicability of ET to quantum-critical models of interacting electrons. We note that a recent additional QMC calculation for the spinfermion model [46], using a different microscopic model from the one in our detailed comparison, also evinces selfenergy behavior consistent with MET.

We used the differences between the theories to identify the importance of high-energy vertex and lattice renormalizations to the low-energy theory. The fact that the bare $\bar{g}$ reproduces both $T_{c}$ and the self-energy well in both models implies that the contributions to fermionic self-energies from vertex corrections due to high-energy fermions are likely small. In addition, we show that MET reproduces the QMC self-energies quite well, but the comparison of QMC with LT shows some systematic deviations. This comparison suggests that there is at least a partial cancellation between vertex corrections and the contribution from highenergy fermions to one-loop self-energy.

\section{SUMMARY}

In this work, we studied the effect of thermal fluctuations on metals near a QCP, either to a spin-density-wave state or to an Ising nematic state. We calculated the deviation from the scaling behavior predicted by ET, in the regime where the thermal contributions do not permit a separation of scales between fermionic and bosonic degrees of freedom. We found that, at low temperatures, close to the QCP, the thermal contribution to fermionic self-energy scales as $\omega_{T} \propto$ $\sqrt{\bar{g}} T$ and dominates the contribution from quantum dynamics. We showed that once this additional physics is taken into account, by an appropriately modified Eliashberg theory, it reproduces properties that were found in recent QMC simulations, above the superconducting $T_{c}$.

In the absence of pair breaking, the regime of metallic quantum critical behavior is limited by the superconducting $T_{c}$ near the QCP and the associated strong superconducting fluctuations. QMC studies report no clear separation between the energy scales associated with superconductivity and non-Fermi-liquid behavior. As we discussed in the Introduction, this result is in agreement with the predictions of ET, where both superconductivity and non-Fermi-liquid behavior appear on a scale of $\omega_{\mathrm{SFM}}, \omega_{\mathrm{INM}}$ for the spinfermion model and Ising-nematic model, respectively. Recent work has indicated [62] that superconducting fluctuations play a significant role in the normal state. Our analysis of the normal-state self-energy neglects superconducting fluctuations, so the combined effect of thermal and superconducting fluctuations remains an open question. We hope our findings will motivate further numerical and analytic work on quantum critical metals.

\section{ACKNOWLEDGMENTS}

We thank S. Lederer, M. H. Christensen, X. Wang, R. M. Fernandes, X.-Y. Xu, K. Sun, Z. Y. Meng, and L. Classen for helpful conversations. This work was supported by the USIsrael Binational Science Foundation (BSF) Grant No. 2018217. E. B. acknowledges support from the European Research Council (ERC) under the HQMAT grant (GrantNo. 817799) and from the MinervaFoundation. Y. S. was 
supported by the Department of Energy, Office of Basic Energy Sciences, under Contract No. DE-AC02-76SF00515 at Stanford, and by the Zuckerman STEM Leadership Program.

\section{APPENDIX: QUANTUM SELF-ENERGY AT THE FIRST MATSUBARA FREQUENCY}

In this Appendix, we explicitly compute the value of the quantum part of the self-energy at the first Matsubara frequency $\Sigma_{Q}(\pi T)$. Our purpose is to justify the somewhat puzzling result, shown in Figs. 5 and 8, that $\Sigma_{Q}(\pi T)$ is negative. We will show that $\Sigma_{Q}(\pi T)$ in MET is always negative and that this is a consequence of the so-called "first Matsubara frequency rule"-namely, that $\Sigma_{Q}(\pi T)=0$ in ET [63,64].

We assume that we are at the QCP $(M=0)$ and write down an expression for $\Sigma_{Q}(\pi T)$ directly from Eq. (18),

$$
\begin{aligned}
\Sigma_{Q}(\pi T) \approx & \bar{g} T \sum_{n \neq 0} \int_{0}^{\infty} \frac{|\mathbf{p}| d p}{2 \pi} \frac{\sigma\left(\Omega_{n}+\pi T\right)}{\sqrt{\left(\Omega_{n}+\pi T+\Sigma\left(\Omega_{n}+\pi T\right)\right)^{2}+v_{F}^{2}|\mathbf{p}|^{2}}} \frac{1}{|\mathbf{p}|^{2}+\Pi\left(|\mathbf{p}|, \Omega_{n}\right)} \\
= & \bar{g} T \sum_{n \geq 1} \int_{0}^{\infty} \frac{|\mathbf{p}| d p}{2 \pi} \frac{1}{|\mathbf{p}|^{2}+\Pi\left(|\mathbf{p}|, \Omega_{n}\right)}\left(\frac{1}{\sqrt{\left(\Omega_{n}+\pi T+\Sigma\left(\Omega_{n}+\pi T\right)\right)^{2}+v_{F}^{2}|\mathbf{p}|^{2}}}\right. \\
& \left.-\frac{1}{\sqrt{\left(\Omega_{n}-\pi T+\Sigma\left(\Omega_{n}-\pi T\right)\right)^{2}+v_{F}^{2}|\mathbf{p}|^{2}}}\right) .
\end{aligned}
$$

For simplicity, we set $v_{F}(\theta)=v_{F}, \nu_{F}(\theta)=\nu_{F}, f(\theta)=1$, and $N_{b}=1$.

It is immediately evident that if we neglect the dynamical part in the square root of the denominator of Eq. (A1), as is done in ET (see Sec. II C), we obtain $\Sigma_{Q}(\pi T)=0$ because contributions from positive and negative frequencies exactly cancel out. This is the first Matsubara frequency rule. The correction coming from MET is therefore coming from the small asymmetry between positive and negative frequencies that arise from the nonfactorization of momentum integration; see the discussion in the Introduction and in Sec. II C. To proceed, we assume and then verify that (i) the integration and summation in Eq. (A1) are dominated by large $v_{F} p, \Omega_{n} \gg \pi T$, and that (ii) we may neglect the self-energy terms. Expanding the square roots, we obtain

$$
\Sigma_{Q}(\pi T) \approx-\frac{\bar{g} T}{2 \pi} \sum_{n \geq 1} \int_{0}^{\infty} \frac{|\mathbf{p}| d p}{|\mathbf{p}|^{2}+\Pi\left(|\mathbf{p}|, \Omega_{n}\right)} \frac{2 \pi T \Omega_{n}}{\left(\Omega_{n}^{2}+(\pi T)^{2}+v_{F}^{2}|\mathbf{p}|^{2}\right)^{3 / 2}}
$$

We first solve for the spin-fermion model. Here, $v_{F}^{2} \Pi^{\mathrm{SFM}}\left(|\mathbf{p}|, \Omega_{n} \mid\right)=\omega_{b} \Omega_{n}$, where $\omega_{b}=\left(\bar{g} N \nu_{F} v_{F} / 2 \pi Q_{h s}\right)$ (by order of magnitude, $\omega_{b} \sim \bar{g}$ ). Rescaling the $p$ integral, we find

$$
\Sigma_{Q}^{\mathrm{SFM}}(\pi T) \approx-\frac{\bar{g} T}{2 \pi} \sum_{n \geq 1} \int_{0}^{\infty} \frac{x d x}{x^{2}+\frac{\omega_{b} \Omega_{n}}{\Omega_{n}^{2}+(\pi T)^{2}}} \frac{2 \pi T \Omega_{n}}{\left(\Omega_{n}^{2}+(\pi T)^{2}\right)^{3 / 2}\left(x^{2}+1\right)^{3 / 2}} .
$$

By inspection, one may verify that the integral is dominated by $x \sim 1, \Omega_{n} \sim \omega_{b}$. Since $\omega_{b}$ is a large parameter in the theory, this result justifies our previous assumptions and also allows us to safely replace the summation with an integral. We then obtain

$$
\begin{aligned}
\Sigma_{Q}^{\mathrm{SFM}}(\pi T) & \approx-\frac{\bar{g} T}{2 \pi} \int_{\pi T}^{\infty} \frac{d \Omega}{\Omega^{2}} \int_{0}^{\infty} \frac{x d x}{\left(x^{2}+\frac{\omega_{b}}{\Omega}\right)\left(x^{2}+1\right)^{3 / 2}} \\
& \approx-\frac{\bar{g} T}{2 \pi \omega_{b}} \log \frac{\omega_{b}}{\pi T} .
\end{aligned}
$$

We see that $\Sigma_{Q}^{\mathrm{SFM}}(\pi T)$ is negative and of order $\bar{g} T / \omega_{b} \sim T$. For other Matsubara frequencies, $\omega_{n}=O(T)$, and $\Sigma_{Q}^{\mathrm{SFM}}\left(\omega_{n}\right)$ is positive and of order $\left(T \omega_{\mathrm{SFM}}\right)^{1 / 2} \sim(T \bar{g})^{1 / 2} \sim T(\bar{g} / T)^{1 / 2}$, i.e., is much larger than $\Sigma_{Q}^{\mathrm{SFM}}(\pi T)$.

For the Ising-nematic model, we write $v_{F}^{2} \Pi^{\mathrm{INM}}\left(|\mathbf{p}|, \Omega_{n}\right)=\omega_{b^{\prime}}^{2}\left(\Omega_{n} / v_{F}|\mathbf{p}|\right)$, where $\omega_{b^{\prime}}^{2}=\bar{g} \nu_{F} v_{F}^{2} / \pi$ [by an order of magnitude $\omega_{b^{\prime}} \sim\left(\bar{g} E_{F}\right)^{1 / 2}$ ]. Repeating the same computational steps, we find 


$$
\Sigma_{Q}^{\mathrm{INM}}(\pi T) \approx-\frac{\bar{g} T}{2 \pi} \sum_{n \geq 1} \int_{0}^{\infty} \frac{x^{2} d x}{x^{3}+\frac{\omega_{b^{\prime}}^{2} \Omega_{n}}{\left(\Omega_{n}^{2}+(\pi T)^{2}\right)^{3 / 2}}} \frac{2 \pi T \Omega_{n}}{\left(\Omega_{n}^{2}+(\pi T)^{2}\right)^{3 / 2}\left(x^{2}+1\right)^{3 / 2}}
$$

Again, we find $x \sim 1, \Omega_{n} \sim \omega_{b^{\prime}}$, justifying our assumptions. Replacing the summation by an integral, we obtain

$$
\begin{aligned}
\Sigma_{Q}^{\mathrm{INM}}(\pi T) & \approx-\frac{\bar{g} T}{2 \pi} \int_{\pi T}^{\infty} \frac{d \Omega}{\Omega^{2}} \int_{0}^{\infty} \frac{x^{2} d x}{\left(x^{3}+\frac{\omega_{b^{\prime}}^{2}}{\Omega^{2}}\right)\left(x^{2}+1\right)^{3 / 2}} \\
& \approx-\frac{\bar{g} T}{4 \sqrt{\pi} \omega_{b^{\prime}}} \Gamma^{2}(3 / 4)
\end{aligned}
$$

and $\Gamma(3 / 4)=1.23$. We see that $\Sigma_{Q}^{\mathrm{INM}}(\pi T)$ is again negative. For the Ising-nematic model, it is of order $T \bar{g} / \omega_{b^{\prime}} \sim T\left(\bar{g} / E_{F}\right)^{1 / 2}$. For other Matsubara frequencies $\omega_{n}=O(T), \quad \Sigma_{Q}^{\mathrm{INM}}\left(\Omega_{n}\right)$ is positive and is of order $\left(T^{2} \bar{g}^{2} / E_{F}\right)^{1 / 3} \sim\left(T\left(\bar{g} / E_{F}\right)^{1 / 2}\right)\left(\bar{g} E_{F} / T^{2}\right)^{1 / 6}$, i.e., is again much larger than $\Sigma_{Q}^{\mathrm{INM}}(\pi T)$.

[1] J. A. Hertz, Quantum Critical Phenomena, Phys. Rev. B 14, 1165 (1976).

[2] A. J. Millis, Effect of a Nonzero Temperature on Quantum Critical Points in Itinerant Fermion Systems, Phys. Rev. B 48, 7183 (1993).

[3] B. L. Altshuler, L. B. Ioffe, and A. J. Millis, Low-Energy Properties of Fermions with Singular Interactions, Phys. Rev. B 50, 14048 (1994).

[4] A. Abanov, A. V. Chubukov, and J. Schmalian, QuantumCritical Theory of the Spin-Fermion Model and Its Application to Cuprates: Normal State Analysis, Adv. Phys. 52, 119 (2003).

[5] A. Abanov, A. V. Chubukov, and A. M. Finkel'stein, Coherent vs. Incoherent Pairing in 2D Systems near Magnetic Instability, Europhys. Lett. 54, 488 (2001).

[6] V. Oganesyan, S. A. Kivelson, and E. Fradkin, Quantum Theory of a Nematic Fermi Fluid, Phys. Rev. B 64, 195109 (2001).

[7] W. Metzner, D. Rohe, and S. Andergassen, Soft Fermi Surfaces and Breakdown of Fermi-Liquid Behavior, Phys. Rev. Lett. 91, 066402 (2003).

[8] M. A. Metlitski and S. Sachdev, Quantum Phase Transitions of Metals in Two Spatial Dimensions. I. Ising-Nematic Order, Phys. Rev. B 82, 075127 (2010).

[9] M. A. Metlitski and S. Sachdev, Quantum Phase Transitions of Metals in Two Spatial Dimensions. II. Spin Density Wave Order,Phys. Rev. B 82, 075128 (2010).

[10] M. A. Metlitski and S. Sachdev, Instabilities near the Onset of Spin Density Wave Order in Metals, New J. Phys. 12, 105007 (2010).

[11] S. Lederer, Y. Schattner, E. Berg, and S. A. Kivelson, Superconductivity and Non-Fermi Liquid Behavior near a Nematic Quantum Critical Point, Proc. Natl. Acad. Sci. U.S.A. 114, 4905 (2017).

[12] S.-S. Lee, Recent Developments in Non-Fermi Liquid Theory, Annu. Rev. Condens. Matter Phys. 9, 227 (2018).

[13] D. L. Maslov and A. V. Chubukov, Fermi Liquid near Pomeranchuk Quantum Criticality, Phys. Rev. B 81, 045110 (2010).
[14] E. Fradkin, S. A. Kivelson, M. J. Lawler, J. P. Eisenstein, and A. P. Mackenzie, Nematic Fermi Fluids in Condensed Matter Physics, Annu. Rev. Condens. Matter Phys. 1, 153 (2010).

[15] Y. Wang, A. Abanov, B. L. Altshuler, E. A. Yuzbashyan, and A. V. Chubukov, Superconductivity near a QuantumCritical Point: The Special Role of the First Matsubara Frequency, Phys. Rev. Lett. 117, 157001 (2016).

[16] S. Raghu, G. Torroba, and H. Wang, Metallic Quantum Critical Points with Finite BCS Couplings, Phys. Rev. B 92 , 205104 (2015).

[17] M. A. Metlitski, D. F. Mross, S. Sachdev, and T. Senthil, Cooper Pairing in Non-Fermi Liquids, Phys. Rev. B 91, 115111 (2015).

[18] S. Lederer, Y. Schattner, E. Berg, and S. A. Kivelson, Enhancement of Superconductivity near a Nematic Quantum Critical Point, Phys. Rev. Lett. 114, 097001 (2015).

[19] Hi. V. Löhneysen, A. Rosch, M. Vojta, and P. Wölfle, Fermi-Liquid Instabilities at Magnetic Quantum Phase Transitions, Rev. Mod. Phys. 79, 1015 (2007).

[20] P. Monthoux, D. Pines, and G. G. Lonzarich, Superconductivity without Phonons, Nature (London) 450, 1177 (2007).

[21] D. J. Scalapino, A Common Thread: The Pairing Interaction for Unconventional Superconductors, Rev. Mod. Phys. 84, 1383 (2012).

[22] S. Sachdev, M. A. Metlitski, and M. Punk, Antiferromagnetism in Metals: From the Cuprate Superconductors to the Heavy Fermion Materials, J. Phys. Condens. Matter 24, 294205 (2012), and references therein.

[23] O. Cyr-Choinière, R. Daou, F. Laliberté, C. Collignon, S. Badoux, D. LeBoeuf, J. Chang, B. J. Ramshaw, D. A. Bonn, W. N. Hardy, R. Liang, J.-Q. Yan, J.-G. Cheng, J.-S. Zhou, J. B. Goodenough, S. Pyon, T. Takayama, H. Takagi, N. Doiron-Leyraud, and L. Taillefer, Pseudogap Temperature $T^{*}$ of Cuprate Superconductors from the Nernst Effect, Phys. Rev. B 97, 064502 (2018).

[24] R. M. Fernandes, A. V. Chubukov, and J. Schmalian, What Drives Nematic Order in Iron-Based Superconductors?, Nat. Phys. 10, 97 (2014). 
[25] F. Wang, S. A. Kivelson, and D.-H. Lee, Nematicity and Quantum Paramagnetism in FeSe, Nat. Phys. 11, 959 (2015).

[26] Y. Wang and A. Chubukov, Charge-Density-Wave Order with Momentum $(2 q, 0)$ and $(0,2 q)$ within the SpinFermion Model: Continuous and Discrete Symmetry Breaking, Preemptive Composite Order, and Relation to Pseudogap in Hole-Doped Cuprates, Phys. Rev. B 90, 035149 (2014).

[27] A. J. Millis, Nearly Antiferromagnetic Fermi Liquids: An Analytic Eliashberg Approach, Phys. Rev. B 45, 13047 (1992).

[28] J. Rech, C. Pépin, and A. V. Chubukov, Quantum Critical Behavior in Itinerant Electron Systems: Eliashberg Theory and Instability of a Ferromagnetic Quantum Critical Point, Phys. Rev. B 74, 195126 (2006).

[29] A. V. Chubukov, A. Abanov, I. Esterlis, and S. A. Kivelson, Eliashberg Theory of Phonon-Mediated SuperconductivityWhen It Is Valid and How It Breaks Down, arXiv:2004.01281.

[30] S.-S. Lee, Low-Energy Effective Theory of Fermi Surface Coupled with $U(1)$ Gauge Field in $2+1$ Dimensions, Phys. Rev. B 80, 165102 (2009).

[31] S-S. Lee, Y. B. Kim, and A. V. Chubukov (unpublished).

[32] T. Holder and W. Metzner, Anomalous Dynamical Scaling from Nematic and U(1) Gauge Field Fluctuations in Two-Dimensional Metals, Phys. Rev. B 92, 041112(R) (2015).

[33] T. Holder and W. Metzner, Fermion Loops and Improved Power-Counting in Two-Dimensional Critical Metals with Singular Forward Scattering, Phys. Rev. B 92, 245128 (2015).

[34] Whether these logarithmic corrections give rise to the appearance of an anomalous fermionic residue but preserve the $\omega^{2 / 3}$ scaling for the self-energy is not known.

[35] It was argued [12] that because of these logarithms, the system eventually flows towards the new fixed point with the dynamical exponent $z=1$.

[36] Y. Wang and A. V. Chubukov, Superconductivity at the Onset of Spin-Density-Wave Order in a Metal, Phys. Rev. Lett. 110, 127001 (2013).

[37] N. E. Bonesteel, I. A. McDonald, and C. Nayak, Gauge Fields and Pairing in Double-Layer Composite Fermion Metals, Phys. Rev. Lett. 77, 3009 (1996).

[38] A. V. Chubukov, P. Monthoux, and D. K. Morr, Vertex Corrections in Antiferromagnetic Spin-Fluctuation Theories, Phys. Rev. B 56, 7789 (1997).

[39] Y. Schattner, M.H. Gerlach, S. Trebst, and E. Berg, Competing Orders in a Nearly Antiferromagnetic Metal, Phys. Rev. Lett. 117, 097002 (2016).

[40] M. H. Gerlach, Y. Schattner, E. Berg, and S. Trebst, Quantum Critical Properties of a Metallic Spin-DensityWave Transition, Phys. Rev. B 95, 035124 (2017).

[41] Y. Schattner, S. Lederer, S. A. Kivelson, and E. Berg, Ising Nematic Quantum Critical Point in a Metal: A Monte Carlo Study, Phys. Rev. X 6, 031028 (2016).

[42] X. Wang, Y. Schattner, E. Berg, and R. M. Fernandes, Superconductivity Mediated by Quantum Critical Antiferromagnetic Fluctuations: The Rise and Fall of Hot Spots, Phys. Rev. B 95, 174520 (2017).
[43] Z. H. Liu, X. Y. Xu, Y. Qi, K. Sun, and Z. Y. Meng, Itinerant Quantum Critical Point with Frustration and a Non-Fermi Liquid, Phys. Rev. B 98, 045116 (2018).

[44] X. Y. Xu, Y. Qi, J. Liu, L. Fu, and Z. Y. Meng, Self-Learning Quantum Monte Carlo Method in Interacting Fermion Systems, Phys. Rev. B 96, 041119(R) (2017).

[45] X. Y. Xu, K. Sun, Y. Schattner, E. Berg, and Z. Y. Meng, Non-Fermi Liquid at $(2+1) \mathrm{D}$ Ferromagnetic Quantum Critical Point, Phys. Rev. X 7, 031058 (2017).

[46] C. Bauer, Y. Schattner, S. Trebst, and E. Berg, Hierarchy of Energy Scales in an $O(3)$ Symmetric Antiferromagnetic Quantum Critical Metal: A Monte Carlo Study, Phys. Rev. Research 2, 023008 (2020).

[47] X. Y. Xu, Z. H. Liu, G. Pan, Y. Qi, K. Sun, and Z. Y. Meng, Revealing Fermionic Quantum Criticality from New Monte Carlo Techniques, J. Phys. Condens. Matter 31, 463001 (2019).

[48] E. Berg, S. Lederer, Y. Schattner, and S. Trebst, Monte Carlo Studies of Quantum Critical Metals, Annu. Rev. Condens. Matter Phys. 10, 63 (2019).

[49] L. Dell'Anna and W. Metzner, Fermi Surface Fluctuations and Single Electron Excitations Near Pomeranchuk Instability in Two Dimensions, Phys. Rev. B 73, 045127 (2006).

[50] H. Yamase and W. Metzner, Fermi-Surface Truncation from Thermal Nematic Fluctuations, Phys. Rev. Lett. 108, 186405 (2012).

[51] M. Punk, Finite-Temperature Scaling Close to Ising-Nematic Quantum Critical Points in Two-Dimensional Metals, Phys. Rev. B 94, 195113 (2016).

[52] H. Wang and G. Torroba, Non-Fermi Liquids at Finite Temperature: Normal-State and Infrared Singularities, Phys. Rev. B 96, 144508 (2017).

[53] A. A. Abrikosov, L. P. Gorkov, and I. E. Dzyaloshinski, Methods of Quantum Field Theory in Statistical Physics, Dover Books on Physics Series (Dover, New York, 1975).

[54] A. V. Chubukov, Ward Identities for Strongly Coupled Eliashberg Theories, Phys. Rev. B 72, 085113 (2005).

[55] A common definition of the thermal contribution to the selfenergy on the real axis is the contribution coming from thermal bosons, i.e., from approximating the Bose function as $n_{B}(\omega) \approx T / \omega$. The definition of $\Sigma_{T}$ we use here is not identical to that one, as can be verified by analytically continuing $\Sigma_{T}\left(\omega_{m}\right)$ to the real axis, but the difference is significant only away from the critical point.

[56] J.-P. Blaizot and E. Iancu, Lifetimes of Quasiparticles and Collective Excitations in Hot QED Plasmas, Phys. Rev. D 55, 973 (1997).

[57] J. A. Damia, M. Solis, and G. Torroba, How Non-Fermi Liquids Cure Their Infrared Divergences, arXiv:2004.05181.

[58] In actuality, it is only exact for $\Omega_{m} / q \rightarrow 0$ or $\Omega_{m} / q \rightarrow \infty$, but because of the constraint imposed at these two limits, the corrections for finite $\Omega_{m} / q$ are at most of order one.

[59] E. O. Tuck, A Simple 'Filon-Trapezoidal' Rule, Math. Comput. 21, 239 (1967).

[60] For $T<0.1$, the QMC data show that the nematic coherence length is already cut off by superconductivity, so we did not use those data.

[61] Note that the asymptotic expression in Eq. (41) for the MET applies when $v_{F} q \ll \omega_{T}$, which is not the case for the QMC 
data. We performed the angular integration in that equation numerically.

[62] A. V. Chubukov, A. Abanov, Y. Wang, and Y.-M. Wu, The Interplay between Superconductivity and Non-Fermi Liquid at a Quantum-Critical Point in a Metal, Ann. Phys. (Amsterdam) 417, 168142 (2020), Eliashberg Theory at 60: Strong-Coupling Superconductivity and Beyond.
[63] A. V. Chubukov and D. L. Maslov, First-MatsubaraFrequency Rule in a Fermi Liquid. I. Fermionic SelfEnergy, Phys. Rev. B 86, 155136 (2012).

[64] An. V. Chubukov and D. L. Maslov, Optical Conductivity of a Two-Dimensional Metal near a Quantum Critical Point: The Status of the Extended Drude Formula, Phys. Rev. B 96, 205136 (2017). 